\title{
Robust Model Predictive Control for Time Delayed Systems with Optimizing Targets and Zone Control
}

\author{
Alejandro H. González ${ }^{1}$ and Darci Odloak ${ }^{2}$ \\ 1 Institute of Technological Development for the Chemical Industry (INTEC), \\ CONICET - Universidad Nacional del Litoral (U.N.L.). \\ Güemes 3450, (3000) Santa Fe, \\ ${ }^{2}$ Department of Chemical Engineering, University of São Paulo, \\ Av. Prof. Luciano Gualberto, tro 3 380, 61548 São Paulo, \\ ${ }^{1}$ Argentina \\ ${ }^{2}$ Brazil
}

\section{Introduction}

Model Predictive Control (MPC) is frequently implemented as one of the layers of a control structure where a Real Time Optimization (RTO) algorithm - laying in an upper layer of this structure - defines optimal targets for some of the inputs and/or outputs (Kassmann et al., 2000). The main scope is to reach the most profitable operation of the process system while preserving safety and product specification constraints. The model predictive controller is expected to drive the plant to the optimal operating point, while minimizing the dynamic error along the input and output paths. Since in the control structure considered here the model predictive controller is designed to track the optimal targets, it is expected that for nonlinear process systems, the linear model included in the controller will become uncertain as we move from the design condition to the optimal condition. The robust MPC presented in this chapter explicitly accounts for model uncertainty of open loop stable systems, where a different model corresponds to each operating point of the process system. In this way, even in the presence of model uncertainty, the controller is capable of maintaining all outputs within feasible zones, while reaching the desired optimal targets. In several other process systems, the aim of the MPC layer is not to guide all the controlled variables to optimal targets, but only to maintain them inside appropriate ranges or zones. This strategy is designated as zone control (Maciejowski, 2002). The zone control may be adopted in some systems, where there are highly correlated outputs to be controlled, and there are not enough inputs to control all the outputs. Another class of zone control problems relates to using the surge capacity of tanks to smooth out the operation of a process unit. In this case, it is desired to let the level of the tank to float between limits, as necessary, to buffer disturbances between sections of a plant. The paper by Qin and Badgwell (2003), which surveys the existing industrial MPC technology, describes a variety of industrial controllers and mention that they always provide a zone control option. Other example of zone control can be found in Zanin et al, (2002), where the authors exemplify the application of this 
strategy in the real time optimization of a FCC system. Although this strategy shows to have an acceptable performance, stability is not usually proved, even when an infinite horizon is used, since the control system keeps switching from one controller to another throughout the continuous operation of the process.

There are several research works that treat the problem of how to obtain a stable MPC with fixed output set points. Although stability of the closed loop is commonly achieved by means of an infinite prediction horizon, the problem of how to eliminate output steady state offset when a supervisory layer produces optimal economic set points, and how to explicitly incorporate the model uncertainty into the control problem formulation for this case, remain an open issue. For the nominal model case, Rawlings (2000), Pannochia and Rawlings (2003), Muske and Badgwell (2002), show how to include disturbance models in order to assure that the inputs and states are led to the desired values without offset. Muske and Badgwell (2002) and Pannochia and Rawlings (2003) develop rank conditions to assure the detectability of the augmented model.

For the uncertain system, Odloak (2004) develops a robust MPC for the multi-plant uncertainty (that is, for a finite set of possible models) that uses a non-increasing cost constraint (Badgwell, 1997). In this strategy, the MPC cost function to be minimized is computed using a nominal model, but the non-increasing cost constraint is settled for each of the models belonging to the set. The stability is then achieved by means of the recursive feasibility of the optimization problem, instead of the optimality. On the other hand, there exist some recent MPC formulations that are based on the existence of a control Lyapunov function (CLF), which is independent of the control cost function. Although the construction of the CFL may not be a trivial task, these formulations also allow the explicit characterization of the stability region subject to constraints and they do not need an infinite output horizon. Mashkar et al. (2006) explore this approach for the control of nominal nonlinear systems, and Mashkar (2006) extends the approach for the case of model uncertainty and control actuator fault. More recently, González et al. (2009) extended the infinite horizon approach to stabilize the closed loop with the MPC controller for the case of multi-model uncertainty and optimizing targets. They developed a robust MPC by adapting the non-increasing cost constraint strategy to the case of zone control of the outputs and it is desirable to guide some of the manipulated inputs to the targets given by a supervisory stationary optimization stage, while maintaining the controlled output in their corresponding zones, taking into account a finite set of possible models. This problem, that seems to interchange an output tracking by an input-tracking formulation, is not trivial, since once the output lies outside the corresponding zone (because of a disturbance, or a change in the output zones), the priority of the controller is again to control the outputs, even if this implies that the input must be settled apart from its targets.

Since in many process systems, mainly from the chemical and petrochemical industries, the process model shows significant time delays, the main contribution of this chapter is to extend the approach of González et al. (2009) to the case of input delayed multi-model systems by introducing minor modifications in the state space model, in such a way that the structure of the control algorithm is preserved. Simulation of a process system of the oil refining industry illustrates the performance of the proposed strategy.

\section{System representation}

Consider a system with $n u$ inputs and $n y$ outputs, and assume for simplicity that the poles relating any input $u_{j}$ to any output $y_{i}$ are non-repeated. To account for the implementation of 
an intuitive MPC formulation, an output prediction oriented model (OPOM) originally presented in Odloak (2004) is adapted here to the case of time delayed systems. Let us designate $\theta_{i, j}$ the time delay between input $u_{j}$ and output $y_{i}$, and define $p>\max _{i, j} \theta_{i, j}$. Then, the state space model considered here is defined as follows:

$$
\begin{aligned}
& x(k+1)=A x(k)+B \Delta u(k) \\
& y(k)=C x(k)
\end{aligned}
$$

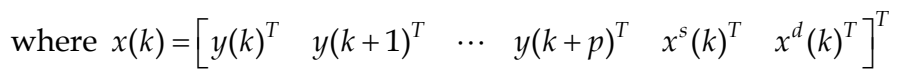

$$
\begin{gathered}
A=\left[\begin{array}{ccccccc}
0 & I_{n y} & 0 & \cdots & 0 & 0 & 0 \\
0 & 0 & I_{n y} & \cdots & 0 & 0 & 0 \\
\vdots & \vdots & & \ddots & \vdots & \vdots & \vdots \\
0 & 0 & 0 & \cdots & I_{n y} & 0 & 0 \\
0 & 0 & 0 & \cdots & 0 & I_{n y} & \Psi((p+1) T) \\
0 & 0 & 0 & \cdots & 0 & I_{n y} & 0 \\
0 & 0 & 0 & \cdots & 0 & 0 & F
\end{array}\right], B=\left[\begin{array}{c}
S_{1} \\
S_{2} \\
\vdots \\
S_{p} \\
S_{p+1} \\
B^{s} \\
B^{d}
\end{array}\right] \\
x^{s} \in \mathfrak{R}^{n y}, x^{d} \in C^{n d}, F \in C^{n d \times n d}, \quad \Psi \in \mathfrak{R}^{n y \times n d}, I_{n y}=\operatorname{diag}\left(\left[\begin{array}{lll}
1 & \cdots & 1
\end{array}\right]\right) \in \mathfrak{R}^{n y \times n y} .
\end{gathered}
$$

The advantage of using the structure of the transition matrix $\mathrm{A}$ is that the state vector is divided into components that are associated to the system modes. In the state equation (1), the state components $x^{s}$ correspond to the (predicted) output steady state, which are in addition the integrating modes of the system (the integrating modes are induced by the incremental form of the inputs), and the components $x^{d}$ correspond to the stable modes of the system. Naturally, when the system approaches steady state these last components tend to zero. For the case of non-repeated pole, $F$ is a diagonal matrix with components of the form $e^{r_{i} T}$ where $r_{i}$ is a pole of the system and $T$ is the sampling period. It is assumed that the system has $n d$ stable poles and $B^{s}$ is the gain matrix of the system. The upper left block of matrix $\mathrm{A}$ is included to account for the time delay of the system. $S_{1}, \ldots, S_{p+1}$ are the step response coefficients of the system. Matrix $\Psi$, which appears in the extended state matrix, is defined as follows

$$
\Psi(t)=\left[\begin{array}{cccc}
\phi_{1}(t) & 0 & \cdots & 0 \\
0 & \phi_{2}(t) & \cdots & 0 \\
\vdots & \vdots & \ddots & \vdots \\
0 & 0 & \cdots & \phi_{n y}(t)
\end{array}\right]
$$

where

$$
\phi_{i}(t)=\left[\begin{array}{lllllll}
e^{r_{i, 1,1}\left(t-\theta_{i, 1}\right)} & \cdots & e^{r_{i, 1, n a}\left(t-\theta_{i, 1}\right)} & \cdots & e^{r_{i, n u, 1}\left(t-\theta_{i, n u}\right)} & \cdots & e^{r_{i, n u, n a}\left(t-\theta_{i, n u}\right)}
\end{array}\right],
$$


$r_{i, j, k}$, with $k=1, \ldots, n a$, are the poles of the transfer function that relates input $u_{j}$ and output $y_{i}$ and $n a$ is the order of this transfer function. It is assumed that $n a$ is the same for any pair $\left(u_{j}, y_{i}\right)$. The time delay affects the dimension of the state matrix $A$ through parameter $p$ and the components of matrix $\Psi$. Input matrix $B$ is also affected by the value of the time delay as the step response coefficients $S_{n}$ will be equal to zero for any $n$ smaller than the time delay.

\subsection{Model uncertainty}

With the model structure presented in (1), model uncertainty is related to uncertainty in matrices $F, B^{\mathrm{s}}, B^{d}$ and the matrix of time delays $\theta$. The uncertainty in these parameters also reflects in the uncertainty of the step response coefficients, which appear in (2). There are several practical ways to represent model uncertainty in model predictive control. One simple way to represent model uncertainty is to consider the multi-plant system (Badgwell, 1997), where we have a discrete set $\Omega$ of plants, and the real plant is unknown, but it is assumed to be one of the components of this set. With this representation of model uncertainty, we can define the set of possible plants as $\Omega=\left\{\Theta_{1}, \ldots, \Theta_{L}\right\}$ where each $\Theta_{n}$ corresponds to a particular plant: $\Theta_{n}=\left(F, B^{s}, B^{d}, \theta\right)_{n}, n=1, \ldots, L$.

Also, let us assume that the true plant, which lies within the set $\Omega$ is designated as $\theta_{T}$ and there is a most likely plant that also lies in $\Omega$ and is designated as $\Theta_{N}$. In addition, it is assumed that the current estimated state corresponds to the true plant.

Badgwell (1997) developed a robust linear quadratic regulator for stable systems with the multi-plant uncertainty. Later, Odloak (2004) extended the method of Badgwell to the output tracking of stable systems considering the same kind of model uncertainty. These strategies include a new constraint corresponding to each of the models lying in $\Omega$, that prevents an increase in the true plant cost function at successive time steps. More recently, González and Odloak (2009) presented an extension of the method by combining the approach presented in Odloak (2004) with the idea of including the output set point as a new restricted optimization variable to develop a robust MPC for systems where the control objective is to maintain the outputs into their corresponding feasible zone, while reaching the desired optimal input target given by the supervisory stationary optimization. In this work the controller proposed by González et al. (2009) is extended to the case of uncertain systems with time delays.

\subsection{System steady state}

As was already said, one of the advantages of the model defined in (1) and (2) is that the state component $x^{s}(k)$ represents the predicted output at steady state, and furthermore this component concentrates the integrating modes of the system. Observe that for the model defined in (1) and (2), if $\Delta u(k+j)=0$ for $j \geq 0$, then the future states can be computed as follows

$$
x(k+j)=A^{j} x(k)
$$

Assuming that $F$ has all the eigenvalues inside the unit circle (i.e. the system is open loop stable), it is easy to show that 


$$
\lim _{j \rightarrow \infty} A^{j}=\left[\begin{array}{ccccc}
0 & 0 & \cdots & I_{n y} & 0 \\
0 & 0 & \cdots & I_{n y} & 0 \\
\vdots & \vdots & \cdots & \vdots & \vdots \\
0 & 0 & \cdots & I_{n y} & 0 \\
0 & 0 & \cdots & 0 & 0
\end{array}\right]
$$

Then, it becomes clear that $\lim _{j \rightarrow \infty} x(k+j)=\left[\begin{array}{llll}x^{s}(k)^{T} & \cdots & x^{s}(k)^{T} & 0\end{array}\right]^{T}$ and consequently $\lim _{j \rightarrow \infty} y(k+j)=C \lim _{j \rightarrow \infty} x(k+j)=x^{s}(k)$. Therefore, $x^{s}(k)$ can be interpreted as the prediction of the output at steady state. The state component $x s(k)$ is assumed to be known or estimated through a stable state observer. A stable observer for the model defined in (1) and (2) is given by

$$
\hat{x}(k+1 \mid k+1)=\hat{x}(k+1 \mid k)+K(y(k+1)-C \hat{x}(k+1 \mid k))
$$

where $K=\left[\begin{array}{llll}I_{n y} & \cdots & I_{n y} & 0\end{array}\right]^{T}$ is the observer gain, and

$$
\begin{gathered}
\hat{x}(k+1 \mid k+1)=\hat{x}(k+1 \mid k)+K(y(k+1)-C \hat{x}(k+1 \mid k)) \\
\hat{x}(k+1 \mid k+1)=(I-K C) A \hat{x}(k \mid k)+(I-K C) B \Delta u(k)
\end{gathered}
$$

For open loop stable systems this is a stable observer as matrix $(I-K C) A$ has the eigenvalues of $F$ and the remaining eigenvalues are equal to zero.

\section{Control structure}

In this work, we consider the control structure shown in Figure 1. In this structure, the economic optimization stage is dedicated to the calculation of the (stationary) desired target, $u_{d e s, k}$, for the input manipulated variables. This stage may be based on a rigorous stationary model and takes into account the process measurements and some economic parameters. In addition, this stage works with a smaller frequency than the low-level control stage, which allows a separation between the two stages. In the zone control framework the low-level control stage, given by the MPC controller, is devoted to guide the manipulated input from the current stationary value $u_{s s}$ to the desired value given by the supervisory economic stage, $u_{d e s, k}$, while keeping the outputs within specified zones. In general, the target $u_{d e s, k}$ will vary whenever the plant operation or the economic parameters change. If it is assumed that the system is currently at a stationary value given by $\left(u_{s s}, y_{s s}\right)$, the desired target $u_{\text {des, } k}$ should satisfy not only the input constraints

$$
u_{\min } \leq u_{d e s, k} \leq u_{\max }
$$

but also the output zone condition

$$
y_{\min } \leq B^{s}\left(\Theta_{n}\right)\left(u_{d e s, k}-u_{s s}\right)+\hat{x}_{n}^{s}(k) \leq y_{\max }, n=1, \ldots, L
$$


where $u_{\min }$ and $u_{\max }$ represent the lower and upper bounds of the input, $y_{\min }$ and $y_{\max }$ represent the lower and upper limits of the output, $B^{S}\left(\Theta_{n}\right)$ is the gain corresponding to a given model $\Theta_{n}$, and $\hat{x}_{n}^{s}(k)$ is the estimated steady-state values of the output corresponding to model $\Theta_{n}$. Note that in the control structure depicted in Figure 1, as the model structure adopted here has integral action, the estimation of component $x_{n}^{s}(k)$ tends to the measured output at steady state for all the models lying in $\Omega$, which means that $\hat{x}_{n}^{s}(k)=y_{s s}$ if the system is at steady state (See González and Odloak 2009 for details). Taking into account this fact, equation (3) can be rewritten as

$$
y_{\min } \leq B^{s}\left(\Theta_{n}\right) u_{d e s, k}+d_{n, s s} \leq y_{\max } \quad n=1, \cdots, L,
$$

where $d_{n, s s}=\hat{x}_{n}^{s}(k)-B^{s}\left(\Theta_{n}\right) u_{s s}=y_{s s}-B^{s}\left(\Theta_{n}\right) u_{s s}$ is the output bias based on the comparison between the current actual output at steady state and the current predicted output at steady state for each model. In other words, $B^{s}\left(\Theta_{n}\right) u_{d e s, k}+d_{n, s s}$ can be interpreted as the corrected output steady state. Note that, since $u_{s s}=\sum_{j=0}^{\bar{k}} \Delta u(j)$, for a large $\bar{k}$, the term $B^{s}\left(\Theta_{n}\right) u_{s s}$ represents the output prediction based only on the past inputs.

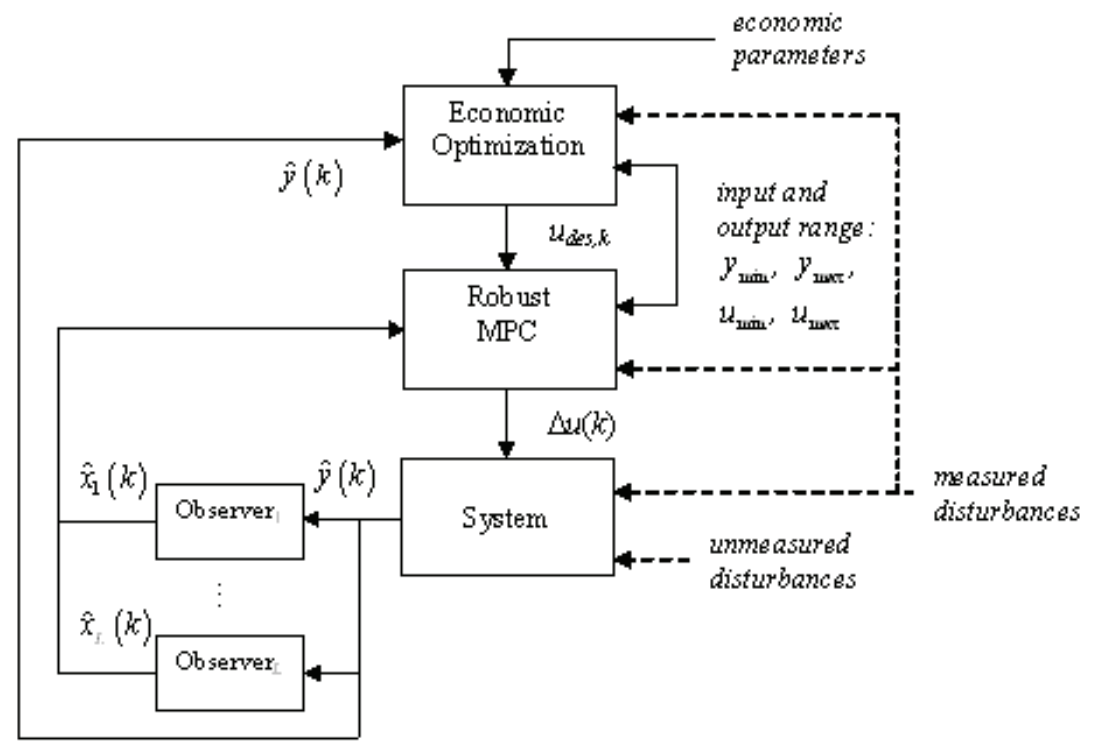

Fig. 1. Control structure.

Based on the later concepts, it is possible to define two input feasible sets for the stationary desired target $u_{d e s, k}$. The first one is the global input feasible set $\vartheta_{o}=\left\{u: u_{\min } \leq u \leq u_{\max }\right\}$, which represents a box-type set. In addition, it is possible to define the more restricted input feasible set $\vartheta_{u}$, which is computed taking into account both, the input constraints and the output limits: 


$$
\vartheta_{u}=\{u: u_{\min } \leq u \leq u_{\max } \text { and } y_{\min } \leq B^{s}\left(\Theta_{n}\right) u-\overbrace{B^{s}\left(\Theta_{n}\right) u_{s s}+y_{s s}}^{d_{n, s s}} \leq y_{\max }, n=1, \cdots, L\} .
$$

This set, which depends on the current stationary point given by $\left(u_{s s}, y_{s s}\right)$, is the intersection of several sets, each one corresponding to a model lying in set $\Omega$. When the output zones are narrow, the restricted input feasible set is smaller than the global feasible set, defined solely by the input constraints. An intuitive diagram of the input feasible set is shown in Figure 4, where three models are used to represent the uncertainty set. In the following sections it will be shown that the proposed controller remains stable and feasible even when the desired input target $u_{\text {des, } k}$ is outside the set $\vartheta_{u}$, or the set $\vartheta_{u}$ itself is null.

\section{Nominal MPC with zone control and input target}

One way to handle the zone control strategy, that is, to maintain the controlled output inside its corresponding range, is by means of an appropriate choice of the output error penalization in the conventional MPC cost function. In this case the output weight is made equal to zero when the system output is inside the range, and the output weight is different from zero if the output prediction is violating any of the constraints, so that the output variable is strictly controlled only if it is outside the feasible range. In this way, the closed loop is guided to a feasible steady state. In Zanin et al. (2002), an algorithm assigns three possible values to the output set points used in the MPC controller: the upper bound of the output feasible range if the predicted output is larger than the upper bound; the lower bound of the output feasible range if the predicted output is smaller than this lower bound; and the predicted output itself, if the predicted output is inside the feasible range. However, a rigorous analysis of the stability of this strategy is not possible even when using an infinite output horizon. González et al. (2006) describe a stable MPC based on the incremental model defined in (1) and (2), that takes into account a stationary optimization of the plant operation. The controller was designed specifically for a heat exchanger network with a number of degrees of freedom larger than zero. In that work, the mismatch between the stationary and the dynamic model was treated by means of an appropriate choice of the weighting matrices in the control cost. However, stability and offset elimination was assured only when the model was perfect.

Based on the work of González et al (2006), we consider the following nominal cost function:

$$
\begin{aligned}
V_{k}= & \sum_{j=0}^{\infty}\left\{\left(y(k+j \mid k)-y_{s p, k}\right)^{T} Q_{y}\left(y(k+j \mid k)-y_{s p, k}\right)+\left(u(k+j \mid k)-u_{d e s, k}\right)^{T} Q_{u}\right. \\
& \left.\left(u(k+j \mid k)-u_{d e s, k}\right)\right\}+\sum_{j=0}^{m-1} \Delta u(k+j \mid k)^{T} R \Delta u(k+j / k)
\end{aligned}
$$

where $\Delta u(k+j \mid k)$ is the control move computed at time $k$ to be applied at time $k+j, m$ is the control or input horizon, $Q_{y}, Q_{u}, R$ are positive weighting matrices of appropriate dimension, $y_{s p, k}$ and $u_{d e s, k}$ are the output and input targets, respectively. The output target $y_{s p, k}$ becomes a computed set point when the output has no optimizing target and consequently the output is controlled by zone. This cost explicitly incorporates an input deviation penalty that tries to accommodate the system at an optimal economic stationary point. 
In the case of systems without time delay the term corresponding to the infinite output error in the cost $V_{k}$ is divided in two parts: the first goes from the current time $k$ to the end of the control horizon, $k+m-1$; while the second one goes from time $k+m$ to infinity. This is so because beyond the control horizon no control actions are implemented and so, considering only the state at time $k+m$, the infinite series can be reduced to a single terminal cost. In the case of time delayed systems, however, the horizon beyond which the entire output evolution can be predicted by a terminal cost is given by $k+p$. As a result, the cost defined in (6) can be developed as follows

$$
\begin{aligned}
V_{k}= & \sum_{j=0}^{p}\left\{\left(y(k+j \mid k)-y_{s p, k}\right)^{T} Q_{y}\left(y(k+j \mid k)-y_{s p, k}\right)\right. \\
+ & \sum_{j=0}^{\infty}\left\{\left(y(k+p+j \mid k)-y_{s p, k}\right)^{T} Q_{y}\left(y(k+p+j \mid k)-y_{s p, k}\right)\right. \\
& \left.\left(u(k+j / k)-u_{d e s, k}\right)^{T} Q_{u}\left(u(k+j / k)-u_{d e s, k}\right)\right\} \\
+ & \sum_{j=0}^{m-1} \Delta u(k+j / k)^{T} R \Delta u(k+j / k)
\end{aligned}
$$

The first term on the right hand side of (7) can be developed as follows

$$
\begin{gathered}
V_{k, 1}=\sum_{j=0}^{p}\left\{\left(y(k+j \mid k)-y_{s p, k}\right)^{T} Q_{y}\left(y(k+j \mid k)-y_{s p, k}\right)\right. \\
V_{k, 1}=\left(\tilde{y}_{k}-\tilde{I}_{y} y_{s p, k}\right)^{T} \tilde{Q}_{y}\left(\tilde{y}_{k}-\tilde{I}_{y} y_{s p, k}\right)
\end{gathered}
$$

where

$$
\begin{aligned}
& \tilde{y}_{k}=N_{x} x(k)+\tilde{S} \Delta u_{k} \\
& \tilde{y}_{k}=\left[\begin{array}{c}
y(k \mid k) \\
y(k+1 \mid k) \\
\vdots \\
y(k+p \mid k)
\end{array}\right], \quad N_{x}=\left[\begin{array}{ll}
I_{(p+1) n y} & 0
\end{array}\right] \in \mathfrak{R}^{(p+1) n y \times n x} ; \tilde{S}=\left[\begin{array}{cccc}
0 & 0 & \cdots & 0 \\
S_{1} & 0 & \cdots & 0 \\
S_{2} & S_{1} & \cdots & 0 \\
\vdots & \vdots & & \vdots \\
S_{p} & S_{p-1} & \cdots & S_{p-m+1}
\end{array}\right] \text {, } \\
& \tilde{I}_{y}=\left[\begin{array}{lll}
I_{n y} & \cdots & I_{n y}
\end{array}\right]^{T}, \tilde{I}_{y} \in \mathfrak{R}^{(p+1) n y \times n y}
\end{aligned}
$$

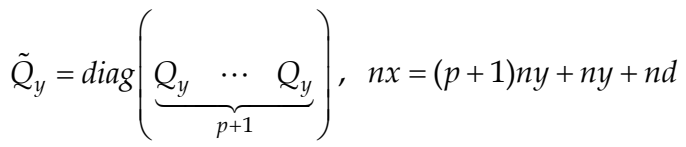

Consequently, considering (8), the term $V_{k, 1}$ can be written as follows 


$$
V_{k, 1}=\left[N_{x} x(k)+\tilde{S} \Delta u_{k}-\tilde{I}_{y} y_{s p, k}\right]^{T} \tilde{Q}_{y}\left[N_{x} x(k)+\tilde{S} \Delta u_{k}-\tilde{I}_{y} y_{s p, k}\right]
$$

The term corresponding to the infinite horizon error on the system output in (7) can be written as follows

$$
\begin{gathered}
V_{k, 2}=\sum_{j=1}^{\infty}\left(y(k+p+j \mid k)-y_{s p, k}\right)^{T} Q_{y}\left(y(k+p+j \mid k)-y_{s p, k}\right) \\
V_{k, 2}=\sum_{j=1}^{\infty}\left(x^{s}(k+m \mid k)+\Psi(p+j-m) x^{d}(k+m \mid k)-y_{s p, k}\right)^{T} Q_{y} \\
\left(x^{s}(k+m \mid k)+\Psi(p+j-m) x^{d}(k+m \mid k)-y_{s p, k}\right)
\end{gathered}
$$

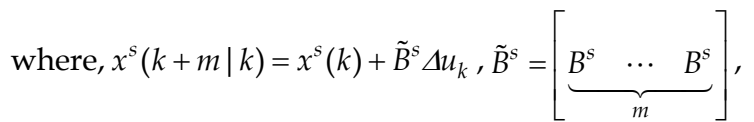

$$
\Delta u_{k}=\left[\begin{array}{lll}
\Delta u(k / k)^{T} & \cdots & \Delta u(k+m-1 / k)^{T}
\end{array}\right]^{T} \in \mathfrak{R}^{m \cdot n u}
$$

Also,

$$
\begin{gathered}
x^{d}(k+m \mid k)=F^{m} x^{d}(k)+\tilde{B}^{d} \Delta u_{k}, \tilde{B}^{d}=\left[\begin{array}{llll}
F^{m-1} B^{d} & F^{m-2} B^{d} & \cdots & B^{d}
\end{array}\right] \\
\Psi(p+j-m)=\Psi(p-m) F^{j}
\end{gathered}
$$

In order to force $V_{k, 2}$ to be bounded, we include the following constraint in the control problem

$$
x^{s}(k+m \mid k)-y_{s p, k}=0 \text { or } x^{s}(k)+\tilde{B}^{s} \Delta u_{k}-y_{s p, k}=0
$$

With the above equation and (11), Eq. (10) becomes

$$
\begin{gathered}
V_{k, 2}=\sum_{j=1}^{\infty}\left(\Psi(p-m) F^{j} x^{d}(k+m \mid k)\right)^{T} Q_{y}\left(\Psi(p-m) F^{j} x^{d}(k+m \mid k)\right) \\
V_{k, 2}=\left(F^{m} x^{d}(k)+\tilde{B}^{d} \Delta u_{k}\right)^{T} Q_{d}\left(F^{m} x^{d}(k)+\tilde{B}^{d} \Delta u_{k}\right)
\end{gathered}
$$

where

$$
Q_{d}=\sum_{j=1}^{\infty}\left(\Psi(p-m) F^{j}\right)^{T} Q_{y}\left(\Psi(p-m) F^{j}\right)
$$

Finally, the infinite term corresponding to the error on the input along the infinite horizon in (7) can be written as follows

$$
V_{k, 3}=\sum_{j=1}^{\infty}\left(u(k+j \mid k)-u_{d e s, k}\right)^{T} Q_{u}\left(u(k+j \mid k)-u_{d e s, k}\right)
$$


Then, it is clear that in order to force (12) to be bounded one needs the inclusion of the following constraint

$$
u(k+m \mid k)-u_{d e s, k}=0
$$

or

$$
u(k-1)+\tilde{I}_{u}^{T} \Delta u_{k}-u_{d e s, k}=0
$$

where $\tilde{I}_{u}^{T}=\left[\begin{array}{lll}\underbrace{I_{n u}}_{m} & \cdots & I_{n u}\end{array}\right]$

Then, assuming that (13) is satisfied, (12) can be written as follows

$V_{k, 3}=\left(\tilde{I}_{u} u(k-1)+M \Delta u_{k}-\tilde{I}_{u} u_{d e s, k}\right)^{T} \tilde{Q}_{u}\left(\tilde{I}_{u} u(k-1)+M \Delta u_{k}-\tilde{I}_{u} u_{d e s, k}\right)$

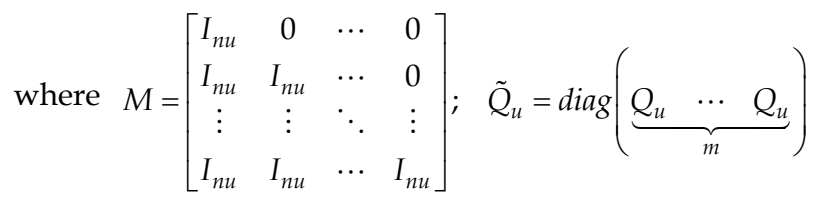

Now, taking into account the proposed terminal constraints, the control cost defined in (7) can be written as follows

$$
\begin{aligned}
V_{k} & =\left[N_{x} x(k)+\tilde{S} \Delta u_{k}-\tilde{I}_{y} y_{s p, k}\right]^{T} \tilde{Q}_{y}\left[N_{x} x(k)+\tilde{S} \Delta u_{k}-\tilde{I}_{y} y_{s p, k}\right] \\
& +\left(F^{m} x^{d}(k)+\tilde{B}^{d} \Delta u_{k}\right)^{T} Q_{d}\left(F^{m} x^{d}(k)+\tilde{B}^{d} \Delta u_{k}\right) \\
& +\left(\tilde{I}_{u} u(k-1)+M \Delta u_{k}-\tilde{I}_{u} u_{d e s, k}\right)^{T} \tilde{Q}_{u}\left(\tilde{I}_{u} u(k-1)+M \Delta u_{k}-\tilde{I}_{u} u_{d e s, k}\right)+\Delta u_{k}^{T} \tilde{R} \Delta u_{k} .
\end{aligned}
$$

To formulate the IHMPC with zone control and input target for the time delayed nominal system, it is convenient to consider the output set point as an additional decision variable of the control problem and the controller results from the solution to the following optimization problem:

subject to

$$
\min _{\Delta u_{k}, y_{s p, k}} V_{k}=\Delta u_{k}^{T} H \Delta u_{k}+2 c_{f}^{T} \Delta u_{k}
$$

$$
\begin{gathered}
u(k-1)+\tilde{I}_{u}^{T} \Delta u_{k}-u_{d e s, k}=0 \\
x^{s}(k)+\tilde{B}^{s} \Delta u_{k}-y_{s p, k}=0 \\
y_{\min } \leq y_{s p, k} \leq y_{\max } \\
-\Delta u_{\max } \leq \Delta u(k+j \mid k) \leq \Delta u_{\max } \quad j=0,1, \cdots, m-1 \\
u_{\min } \leq u(k-1)+\sum_{i=0}^{j} \Delta u(k+i \mid k) \leq u_{\max } ; \quad j=0,1, \cdots, m-1
\end{gathered}
$$


where

$$
\begin{gathered}
H=\tilde{S}^{T} \tilde{Q}_{y} \tilde{S}+\tilde{B}^{d T} Q_{d} \tilde{B}^{d}+M^{T} \tilde{Q}_{u} M+\tilde{R} \\
c_{f}^{T}=x(k)^{T} N_{x}^{T} \tilde{Q}_{y} \tilde{S}+x^{d}(k)^{T}\left(F^{m}\right)^{T} Q_{d} \tilde{B}^{d}+\left(u(k-1)-u_{d e s}\right)^{T} \tilde{I}_{u}^{T} \tilde{Q}_{u} M
\end{gathered}
$$

Constraints (14) and (15) are terminal constraints, and they mean that both, the input and the integrating component of the output errors will be null at the end of the control horizon $m$. Constraint (16), on the other hand, forces the new decision variable $y_{s p, k}$ to be inside the zone given by $y_{\min }$ and $y_{\max }$. So, as $y_{s p, k}$ is a set point variable, constraint (16) means that the effective output set point of the proposed controller is now the complete feasible zone. Notice that if the output bounds are settled so that the upper bound equals the lower bound, then the problem becomes the traditional set point tracking problem.

\subsection{Enlarging the feasible region}

The set of constraints added to the optimization problem in the last section may produce a severe reduction in the feasible region of the resulting controller. Specifically, since the input increments are usually bounded, the terminal constraints frequently result in infeasible problems, which means that it is not possible for the controller to achieve the constraints in $m$ time steps, given that $m$ is frequently small to reduce the computational cost. A possible solution to this problem is to incorporate slack variables in the terminal constraints. So, assuming that the slack variables are unconstrained, it is possible to guarantee that the control problem will be feasible. Besides, these slack variables must be penalized in the cost function with large weights to assure the constraint violation will be minimized by the control actions. Thus, the cost function can be written as follows

$$
\begin{aligned}
V_{k}= & \sum_{j=0}^{p}\left(y(k+j \mid k)-y_{s p, k}-\delta_{y, k}\right)^{T} Q_{y}\left(y(k+j \mid k)-y_{s p, k}-\delta_{y, k}\right) \\
& +\sum_{j=1}^{\infty}\left(y(k+p+j \mid k)-y_{s p, k}-\delta_{y, k}\right)^{T} Q_{y}\left(y(k+p+j \mid k)-y_{s p, k}-\delta_{y, k}\right)+ \\
& +\sum_{j=0}^{m-1}\left(u(k+j \mid k)-u_{d e s, k}-\delta_{u, k}\right)^{T} Q_{u}\left(u(k+j \mid k)-u_{d e s, k}-\delta_{u, k}\right) \\
& +\sum_{j=0}^{\infty}\left(u(k+m+j \mid k)-u_{d e s, k}-\delta_{u, k}\right)^{T} Q_{u}\left(u(k+m+j \mid k)-u_{d e s, k}-\delta_{u, k}\right)+ \\
& +\sum_{j=0}^{m-1} \Delta u(k+j \mid k)^{T} R \Delta u(k+j \mid k)+\delta_{y, k}^{T} S_{y} \delta_{y, k}+\delta_{u, k}^{T} S_{u} \delta_{u, k}
\end{aligned}
$$

where $S_{y}, S_{u}$ are positive definite matrices of appropriate dimension and $\delta_{y, k} \in \mathfrak{R}^{n y}, \delta_{u, k} \in \mathfrak{R}^{n u}$ are the slack variables (new decision variables) that eliminate any infeasibility of the control problem. Following the same steps as in the controller where slacks are not considered, it can be shown that the cost defined in (17) will be bounded if the following constraints are included in the control problem:

$$
x^{s}(k)+\tilde{B}^{s} \Delta u_{k}-y_{s p, k}-\delta_{y, k}=0
$$




$$
u(k-1)+\tilde{I}_{u}^{T} \Delta u_{k}-u_{d e s, k}-\delta_{u, k}=0
$$

In this case, the cost defined in (17) can be reduced to the following quadratic function

$$
\begin{aligned}
V_{k}=\left[\begin{array}{llll}
\Delta u_{k}^{T} & y_{s p, k}^{T} & \delta_{y, k}^{T} & \delta_{u, k}^{T}
\end{array}\right]\left[\begin{array}{cccc}
H_{11} & H_{12} & H_{13} & H_{14} \\
H_{21} & H_{22} & H_{23} & 0 \\
H_{31} & H_{32} & H_{33} & 0 \\
H_{41} & 0 & 0 & H_{44}
\end{array}\right]\left[\begin{array}{c}
\Delta u_{k} \\
y_{s p, k} \\
\delta_{y, k} \\
\delta_{u, k}
\end{array}\right]+ \\
+2\left[\begin{array}{llll}
c_{f, 1} & c_{f, 2} & c_{f, 3} & c_{f, 4}
\end{array}\right]\left[\begin{array}{c}
\Delta u_{k} \\
y_{s p, k} \\
\delta_{y, k} \\
\delta_{u, k}
\end{array}\right]+c
\end{aligned}
$$

where

$$
\begin{gathered}
H_{11}=\tilde{S}^{T} \tilde{Q}_{y} \tilde{S}+\left(\tilde{B}^{d}\right)^{T} Q_{d} \tilde{B}^{d}+M^{T} \tilde{Q}_{u} M+\tilde{R} \\
H_{12}=H_{21}^{T}=-\tilde{S}^{T} \tilde{Q}_{y} \tilde{I}_{y}, H_{13}=H_{31}^{T}=-\tilde{S}^{T} \tilde{Q}_{y} \tilde{I}_{y}, \quad H_{14}=H_{41}^{T}=-M^{T} \tilde{Q}_{u} \tilde{I}_{u} \\
H_{22}=\tilde{I}_{u}^{T} \tilde{Q}_{u} \tilde{I}_{u}, H_{23}=H_{32}^{T}=\tilde{I}_{y}^{T} \tilde{Q}_{y} \tilde{I}_{y}, H_{33}=\tilde{I}_{y}^{T} \tilde{Q}_{y} \tilde{I}_{y}+S_{y}, H_{44}=\tilde{I}_{u}^{T} \tilde{Q}_{u} \tilde{I}_{u}+S_{u} \\
H_{24}=H_{42}^{T}=H_{34}=H_{43}^{T}=0 \\
c_{f, 1}=x(k)^{T} N_{x}^{T} \tilde{Q}_{y} \tilde{S}+x^{d}(k)^{T}\left(F^{m}\right)^{T} Q_{d} B_{m}^{d}+\left(u(k-1)-u_{d e s}\right)^{T} \tilde{I}_{u}^{T} \tilde{Q}_{u} M \\
c_{f, 2}=-x(k)^{T} N_{x}^{T} \tilde{Q}_{y} \tilde{I}_{y}, \quad c_{f, 3}=-x(k)^{T} N_{x}^{T} \tilde{Q}_{y} \tilde{I}_{y} \\
c_{f, 4}=-\left(u(k-1)-u_{d e s, k}\right)^{T} \tilde{I}_{u}^{T} \tilde{Q}_{u} \tilde{I}_{u} \\
c=x(k)^{T} N_{x}^{T} \tilde{Q}_{y} N_{x} x(k)+x^{d}(k)^{T}\left(F^{m}\right)^{T} Q_{d} F^{m} x^{d}(k)+\left(u(k-1)-u_{d e s, k}\right)^{T} \tilde{I}_{u}^{T} \tilde{Q}_{u} \tilde{I}_{u}\left(u(k-1)-u_{d e s, k}\right)
\end{gathered}
$$

Then, the nominally stable MPC controller with guaranteed feasibility for the case of output zone control of time delayed systems with input targets results from the solution to the following optimization problem:

Problem P1

$$
\min _{\substack{\Delta u_{k}, y_{s p, k}, \delta_{y, k}, \delta_{u, k}}} V_{k}
$$

subject to:

$$
-\Delta u_{\max } \leq \Delta u(k+j \mid k) \leq \Delta u_{\max } \quad j=0,1, \cdots, m-1
$$




$$
\begin{gathered}
u_{\min } \leq u(k-1)+\sum_{i=0}^{j} \Delta u(k+i \mid k) \leq u_{\max } ; \quad j=0,1, \cdots, m-1 \\
y_{\min } \leq y_{s p, k} \leq y_{\max } \\
x^{s}(k)+\tilde{B}^{s} \Delta u_{k}-y_{s p, k}-\delta_{y, k}=0 \quad\left(x^{s}(k+m \mid k)-y_{s p, k}-\delta_{y, k}=0\right) \\
u(k-1)+\tilde{I}_{u}^{T} \Delta u_{k}-u_{d e s, k}-\delta_{u, k}=0 \quad\left(u(k+m-1 \mid k)-u_{d e s, k}-\delta_{u, k}=0\right)
\end{gathered}
$$

It must be noted that the use of slack variables is not only convenient to avoid dynamic feasibility problems, but also to prevent stationary feasibility problems. Stationary feasibility problems are usually produced by the supervisory optimization level shown in the control structure defined in Figure 1. In such a case, for instance, the slack variable $\delta_{y, k}$ allows the predicted output to be different from the set point variable $y_{s p, k}$ at steady state (notice that only $y_{s p, k}$ is constrained to be inside the desired zone). So, the slacked problem formulation allows the system output to remain outside the desired zone, if no stationary feasible solution can be found.

It can be shown that the controller produced through the solution of problem P1 results in a stable closed loop system for the nominal system. However, the aim here is to extend this formulation to the case of multi model uncertainty.

\section{Robust MPC with zone control and input target}

In the model formulation presented in (1) and (2) for the time delayed system, uncertainty concentrates not only on matrices $F, B^{s}$ and $B^{d}$ as in the system without time delay, but also on matrix $\theta \in \mathfrak{R}^{n y \times n u}$ that contains all the time delays between the system inputs and outputs. Observe that the step response coefficients $S_{1}, \ldots, S_{p+1}$, which appears in the input matrix and $\Psi(p+1)$, which appears in the state matrix of the model defined in (1) and (2) are also uncertain, but can be computed from $F, B^{s}, B^{d}$ and $\theta$. Now, considering the multimodel uncertainty, assume that each model is designated by a set of parameters defined as $\Theta_{n}=\left\{B_{n}^{s}, B_{n}^{d}, F_{n}, \theta_{n}\right\}, n=1, \ldots, L$. Also, assume that in this case $p>\max \theta_{n}(i, j)+m$ (this condition guarantees that the state vector of all models have the same dimension). Then, for each model $\Theta_{n}$, we can define a cost function as follows

$$
\begin{aligned}
V_{k}\left(\Theta_{n}\right)= & \sum_{j=0}^{p}\left(y_{n}(k+j \mid k)-y_{s p, k}\left(\Theta_{n}\right)-\delta_{y, k}\left(\Theta_{n}\right)\right)^{T} Q_{y}\left(y_{n}(k+j \mid k)-y_{s p, k}\left(\Theta_{n}\right)-\delta_{y, k}\left(\Theta_{n}\right)\right) \\
& +\sum_{j=1}^{\infty}\left(y_{n}(k+p+j \mid k)-y_{s p, k}\left(\Theta_{n}\right)-\delta_{y, k}\left(\Theta_{n}\right)\right)^{T} Q_{y}\left(y_{n}(k+p+j \mid k)-y_{s p, k}\left(\Theta_{n}\right)-\delta_{y, k}\left(\Theta_{n}\right)\right) \\
& +\sum_{j=0}^{m-1}\left(u(k+j \mid k)-u_{d e s, k}-\delta_{u, k}\right)^{T} Q_{u}\left(u(k+j \mid k)-u_{d e s, k}-\delta_{u, k}\right) \\
& +\sum_{j=0}^{\infty}\left(u(k+m+j \mid k)-u_{d e s, k}-\delta_{u, k}\right)^{T} Q_{u}\left(u(k+m+j \mid k)-u_{d e s, k}-\delta_{u, k}\right) \\
& +\sum_{j=0}^{m-1} \Delta u(k+j \mid k)^{T} R \Delta u(k+j \mid k)+\delta_{y, k}^{T}\left(\Theta_{n}\right) S_{y} \delta_{y, k}\left(\Theta_{n}\right)+\delta_{u, k}{ }^{T} S_{u} \delta_{u, k}
\end{aligned}
$$


Following the same steps as in case of the nominal system, we can conclude that the cost defined in (20) will be bounded if the control actions, set points and slack variables are such that (18) is satisfied and

$$
x^{s}(k)+\tilde{B}^{s}\left(\Theta_{n}\right) \Delta u_{k}-y_{s p, k}\left(\Theta_{n}\right)-\delta_{y, k}\left(\Theta_{n}\right)=0
$$

Then, if these conditions are satisfied, (20) can be written as follows

$$
\begin{aligned}
& V_{k}\left(\Theta_{n}\right)=\left(N_{x} x(k)+\tilde{S}\left(\Theta_{n}\right) \Delta u_{k}-\tilde{I}_{y} y_{s p, k}\left(\Theta_{n}\right)-\tilde{I}_{y} \delta_{y, k}\left(\Theta_{n}\right)\right)^{T} \tilde{Q}_{y} \\
& \left(N_{x} x(k)+\tilde{S}\left(\Theta_{n}\right) \Delta u_{k}-\tilde{I}_{y} y_{s p, k}\left(\Theta_{n}\right)-\tilde{I}_{y} \delta_{y, k}\left(\Theta_{n}\right)\right) \\
& +\left(F\left(\Theta_{n}\right)^{m} x^{d}(k)+B_{m}^{d}\left(\Theta_{n}\right) \Delta u_{k}\right)^{T} Q_{d}\left(\Theta_{n}\right)\left(F\left(\Theta_{n}\right)^{m} x^{d}(k)+B_{m}^{d}\left(\Theta_{n}\right) \Delta u_{k}\right) \\
& +\left(\tilde{I}_{u} u(k-1)+M \Delta u_{k}-\tilde{I}_{u} u_{d e s, k}-\tilde{I}_{u} \delta_{u, k}\right)^{T} Q_{u}\left(\tilde{I}_{u} u(k-1)+M \Delta u_{k}-\tilde{I}_{u} u_{d e s, k}-\tilde{I}_{u} \delta_{u, k}\right) \\
& +\Delta u_{k}^{T} \tilde{R} \Delta u_{k}+\delta_{y, k}\left(\Theta_{n}\right)^{T} S_{y} \delta_{y, k}\left(\Theta_{n}\right)+\delta_{u, k}{ }^{T} S_{u} \delta_{u, k}
\end{aligned}
$$

or

$$
\begin{aligned}
& V_{k}\left(\Theta_{n}\right)=\left[\begin{array}{llll}
\Delta u_{k}^{T} & y_{s p, k}^{T}\left(\Theta_{n}\right) & \delta_{y, k}^{T}\left(\Theta_{n}\right) & \delta_{u, k}^{T}
\end{array}\right]\left[\begin{array}{cccc}
H_{11}\left(\Theta_{n}\right) & H_{12}\left(\Theta_{n}\right) & H_{13}\left(\Theta_{n}\right) & H_{14} \\
H_{21}\left(\Theta_{n}\right) & H_{22} & H_{23} & 0 \\
H_{31}\left(\Theta_{n}\right) & H_{32} & H_{33} & 0 \\
H_{41} & 0 & 0 & H_{44}
\end{array}\right]\left[\begin{array}{c}
\Delta u_{k} \\
y_{s p, k}\left(\Theta_{n}\right) \\
\delta_{y, k}\left(\Theta_{n}\right) \\
\delta_{u, k}
\end{array}\right] \\
& +2\left[\begin{array}{llll}
c_{f, 1}\left(\Theta_{n}\right) & c_{f, 2} & c_{f, 3} & c_{f, 4}
\end{array}\right]\left[\begin{array}{c}
\Delta u_{k} \\
y_{s p, k}\left(\Theta_{n}\right) \\
\delta_{y, k}\left(\Theta_{n}\right) \\
\delta_{u, k}
\end{array}\right]+c\left(\Theta_{n}\right) \\
& H_{11}\left(\Theta_{n}\right)=\tilde{S}\left(\Theta_{n}\right)^{T} \tilde{Q}_{y} \tilde{S}\left(\Theta_{n}\right)+\left(\tilde{B}^{d}\left(\Theta_{n}\right)\right)^{T} Q_{d}\left(\Theta_{n}\right) \tilde{B}^{d}\left(\Theta_{n}\right)+M^{T} \tilde{Q}_{u} M+\tilde{R} \\
& H_{12}=H_{21}^{T}=-\tilde{S}\left(\Theta_{n}\right)^{T} \tilde{Q}_{y} \tilde{I}_{y}, \quad H_{13}=H_{31}^{T}=-\tilde{S}\left(\Theta_{n}\right)^{T} \tilde{Q}_{y} \tilde{I}_{y}, \quad H_{14}=H_{41}^{T}=-M^{T} \tilde{Q}_{u} \tilde{I}_{u} \\
& H_{22}=\tilde{I}_{y}^{T} \tilde{Q}_{y} \tilde{I}_{y}, \quad H_{23}=H_{32}^{T}=\tilde{I}_{y}^{T} \tilde{Q}_{y} \tilde{I}_{y}, \quad H_{33}=\tilde{I}_{y}^{T} \tilde{Q}_{y} \tilde{I}_{y} \\
& H_{24}=H_{42}^{T}=H_{34}=H_{43}^{T}=0 \\
& c_{f, 1}=x(k)^{T} N_{x}^{T} \tilde{Q}_{y} \tilde{S}\left(\Theta_{n}\right)+x^{d}(k)^{T}\left(F\left(\Theta_{n}\right)^{m}\right)^{T} Q_{x d} \tilde{B}^{d}\left(\Theta_{n}\right)+\left(u(k-1)-u_{d e s}\right)^{T} \tilde{I}_{u}^{T} \tilde{Q}_{u} M \\
& c_{f, 2}=-x(k)^{T} N_{x}^{T} \tilde{Q}_{y} \tilde{I}_{y}, \quad c_{f, 3}=-x(k)^{T} N_{x}^{T} \tilde{Q}_{y} \tilde{I}_{y} \\
& c_{f, 4}=-\left(u(k-1)-u_{d e s}\right)^{T} \tilde{I}_{u}^{T} \tilde{Q}_{u} \tilde{I}_{u}
\end{aligned}
$$




$$
\begin{aligned}
c & =x(k)^{T} N_{x}^{T} \tilde{Q}_{y} N_{x} x(k)+x^{d}(k)^{T}\left(F\left(\Theta_{n}\right)^{m}\right)^{T} Q_{x d} F\left(\Theta_{n}\right)^{m} x^{d}(k)+ \\
& +\left(u(k-1)-u_{d e s, k}\right)^{T} \tilde{I}_{u}^{T} \tilde{Q}_{u} \tilde{I}_{u}\left(u(k-1)-u_{d e s, k}\right)
\end{aligned}
$$

Then, the robust MPC for the system with time delay and multi-model uncertainty is obtained from the solution to the following problem:

Problem P2

$$
\min _{\substack{\Delta u_{k}, y_{s p, k}\left(\Theta_{n}\right), \delta_{y, k}\left(\Theta_{n}\right), \delta_{u, k} \\ n=1, \ldots, L}} V_{k}\left(\Theta_{N}\right)
$$

subject to

$$
\begin{gathered}
-\Delta u_{\max } \leq \Delta u(k+j \mid k) \leq \Delta u_{\max } \quad j=0,1, \cdots, m-1 \\
u_{\min } \leq u(k-1)+\sum_{i=0}^{j} \Delta u(k+i \mid k) \leq u_{\max } ; \quad j=0,1, \cdots, m-1 \\
y_{\min } \leq y_{s p, k}\left(\Theta_{n}\right) \leq y_{\max } ; \quad n=1, \cdots, L \\
x^{s}(k)+\tilde{B}^{s}\left(\Theta_{n}\right) \Delta u_{k}-y_{s p, k}\left(\Theta_{n}\right)-\delta_{y, k}\left(\Theta_{n}\right)=0 ; \quad n=1, \cdots, L \\
u(k-1)+\tilde{I}_{u}^{T} \Delta u_{k}-u_{d e s, k}-\delta_{u, k}=0 \\
V_{k}\left(\Delta u_{k}, \delta_{y, k}\left(\Theta_{n}\right), \delta_{u, k}, y_{s p, k}\left(\Theta_{n}\right), \Theta_{n}\right) \leq \tilde{V}_{k}\left(\Delta \tilde{u}_{k}, \tilde{\delta}_{y, k}\left(\Theta_{n}\right), \tilde{\delta}_{u, k}, y_{s p, k}\left(\Theta_{n}\right), \Theta_{n}\right), \quad n=1, \cdots, L
\end{gathered}
$$

where, assuming that $\left(\Delta u_{k-1}^{*}, y_{s p, k-1}\left(\Theta_{n}\right), \delta_{u, k-1}^{*}, \delta_{y, k-1}^{*}\left(\Theta_{n}\right)\right)$ is the optimal solution to Problem P2 at time step $k-1$, we define

$\Delta \tilde{u}_{k}=\left[\begin{array}{llll}\Delta u^{*}(k \mid k-1)^{T} & \cdots & \Delta u^{*}(k+m-2 \mid k-1)^{T} & 0\end{array}\right]^{T} ; \quad \tilde{y}_{s p, k}\left(\Theta_{n}\right)=y_{s p, k-1}^{*}\left(\Theta_{n}\right)$ and $\tilde{\delta}_{u, k}$ such that

$$
u(k-1)+\tilde{I}_{u}^{T} \Delta \tilde{u}_{k}-u_{d e s, k}-\tilde{\delta}_{u, k}=0
$$

and define $\tilde{\delta}_{y, k}\left(\Theta_{n}\right)$ such that

$$
x^{s}(k)+\tilde{B}^{s}\left(\Theta_{n}\right) \Delta \tilde{u}_{k}-\tilde{y}_{s p, k}\left(\Theta_{n}\right)-\tilde{\delta}_{y, k}\left(\Theta_{n}\right)=0
$$

In (20), $\Theta_{N}$ corresponds to the nominal or most probable model of the system.

Remark 1: The cost to be minimized in problem P2 corresponds to the nominal model. However, constraints (23) and (24) are imposed considering the estimated state of each model $\Theta_{n} \in \Omega$. Constraint (25) is a non-increasing cost constraint that assures the convergence of the true state cost to zero.

Remark 2: The introduction of $L$ set-point variables allows the simultaneous zeroing of all the output slack variables. In that case, whenever possible, the set-point variable $y_{s p, k}\left(\Theta_{n}\right)$ 
will be equal to the output prediction at steady state (represented by $x_{n}^{s}(k+m)$ ), and so the corresponding output penalization will be removed from the cost. As a result, the controller gains some flexibility that allows achieving the other control objectives.

Remark 3: Note that by hypothesis, one of the observers is based on the actual plant model, and if the initial and the final steady states are known, then the estimated state $\hat{x}_{T}(k)$ will be equal to the actual plant state at each time $k$.

Remark 4: Conditions (26) and (27) are used to update the pseudo variables of constraint (25), by taking into account the current state estimation $\hat{x}_{n}^{s}(k)$ for each of the models lying in $\Omega$, and the last value of the input target.

One important feature that should have a constrained controller is the recursive feasibility (i.e. if the optimization problem is feasible at a given time step, it should remain feasible at any subsequent time step). The following lemma shows how the proposed controller achieves this property.

Lemma. If problem P2 is feasible at time step $k$, it will remain feasible at any subsequent time step $k+j, j=1,2, \ldots$

Proof:

Assume that the output zones remain fixed, and also assume that

$$
\begin{gathered}
\Delta u_{k}^{*}=\left[\begin{array}{lll}
\Delta u^{*}(k \mid k)^{T} & \cdots & \Delta u^{*}(k+m-1 \mid k)^{T}
\end{array}\right]^{T} \in \mathfrak{R}^{m \cdot n u}, \\
y_{s p, k}^{*}\left(\Theta_{1}\right), \cdots, y_{s p, k}^{*}\left(\Theta_{L}\right), \delta_{y, k}^{*}\left(\Theta_{1}\right), \cdots, \delta_{y, k}^{*}\left(\Theta_{L}\right) \text { and } \delta_{u, k}^{*}
\end{gathered}
$$

correspond to the optimal solution to problem P2 at time $k$.

Consider now the pseudo variables $\left(\Delta \tilde{u}_{k+1}, \tilde{y}_{s p, k+1}\left(\Theta_{1}\right), \cdots, \tilde{y}_{s p, k+1}\left(\Theta_{L}\right), \quad \tilde{\delta}_{y, k+1}\left(\Theta_{1}\right), \ldots\right.$, $\left.\tilde{\delta}_{y, k+1}\left(\Theta_{L}\right), \tilde{\delta}_{u, k+1}\right)$ where

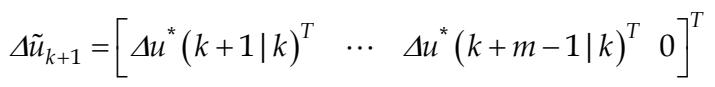

$$
\begin{aligned}
& \tilde{y}_{s p, k+1}\left(\Theta_{n}\right)=y_{s p, k}^{*}\left(\Theta_{n}\right), n=1, \cdots, L,
\end{aligned}
$$

Also, the slacks $\tilde{\delta}_{u, k+1}$ and $\tilde{\delta}_{y, k+1}\left(\Theta_{n}\right)$ are such that

and

$$
u(k)+\tilde{I}_{u}^{T} \Delta \tilde{u}_{k+1}-u_{d e s, k}-\tilde{\delta}_{u, k+1}=0
$$

$$
\hat{x}_{n}^{s}(k+1)+\tilde{B}^{s}\left(\Theta_{n}\right) \Delta \tilde{u}_{k+1}-\tilde{y}_{s p, k+1}\left(\Theta_{n}\right)-\tilde{\delta}_{y, k+1}\left(\Theta_{n}\right)=0, \quad n=1, \ldots, L
$$

We can show that the solution defined through (30) to (33) represent a feasible solution to problem P2 at time $k+1$, which proves the recursive feasibility. This means that if problem $\mathrm{P} 2$ is feasible at time step $k$, then, it will remain feasible at all the successive time steps $k+1$, $k+2, \ldots \square$

Now, the convergence of the closed loop system with the robust controller resulting from the later optimization problem can be stated as follows: 
Theorem. Suppose that the undisturbed system starts at a known steady state and one of the state observers is based on the actual model of the plant. Consider also that the input target is moved to a new value, or the boundaries of the output zones are modified. Then, if condition (3) is satisfied for each model $\Theta_{n} \in \Omega$, the cost function of the undisturbed true system in closed loop with the controller defined through the solution to problem P2 will converge to zero.

Proof:

Suppose that, at time $k$ the uncertain system starts from a steady state corresponding to output $y(k)=y_{s s}$ and input $u(k-1)=u_{s s}$. We have already shown that, with the model structure considered in (1) and (2), the model states corresponding to this initial steady state can be represented as follows:

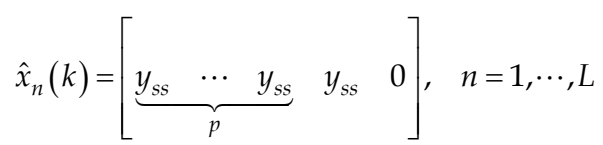

and consequently, $\hat{x}_{n}^{s}(k)=y_{s s}, \quad \hat{x}_{n}^{d}(k)=0, \quad n=1, \cdots, L$.

At time $k$, the cost corresponding to the solution defined in (28) and (29) for the true model is given by

$$
\begin{aligned}
V_{k}^{*}\left(\Theta_{T}\right)= & \sum_{j=0}^{\infty}\left\{\left(y_{T}^{*}(k+j \mid k)-y_{s p, k}^{*}\left(\Theta_{T}\right)-\delta_{y, k}^{*}\left(\Theta_{T}\right)\right)^{T} Q_{y}\left(y_{T}^{*}(k+j \mid k)-y_{s p, k}^{*}\left(\theta_{T}\right)-\delta_{y, k}^{*}\left(\theta_{T}\right)\right)\right. \\
& \left.+\left(u^{*}(k+j \mid k)-u_{d e s, k}-\delta_{u, k}^{*}\right)^{T} Q_{u}\left(u^{*}(k+j \mid k)-u_{d e s, k}-\delta_{u, k}^{*}\right)\right\} \\
& +\sum_{j=0}^{m-1} \Delta u^{*}(k+j \mid k)^{T} R \Delta u^{*}(k+j \mid k)+\delta_{y, k}^{*}{ }^{T}\left(\Theta_{T}\right) S_{y} \delta_{y, k}^{*}\left(\Theta_{T}\right)+\delta_{u, k}^{*} S_{u} \delta_{u, k}^{*}
\end{aligned}
$$

At time step $k+1$, the cost corresponding to the pseudo variables defined in (30) to (33) for the true model is given by

$$
\begin{aligned}
& \ddot{V}_{k+1}\left(\Theta_{T}\right)= \\
& \sum_{j=0}^{\infty}\left\{\left(y_{T}^{*}(k+j+1 \mid k)-y_{s p, k}^{*}\left(\Theta_{T}\right)-\delta_{y, k}^{*}\left(\Theta_{T}\right)\right)^{T} Q_{y}\left(y_{T}^{*}(k+j+1 \mid k)-y_{s p, k}^{*}\left(\Theta_{T}\right)-\delta_{y, k}^{*}\left(\Theta_{T}\right)\right)\right. \\
& \left.\quad+\left(u^{*}(k+j+1 / k)-u_{d e s, k}-\delta_{u, k}^{*}\right)^{T} Q_{u}\left(u^{*}(k+j+1 / k)-u_{d e s, k}-\delta_{u, k}^{*}\right)\right\} \\
& +\sum_{j=0}^{m-1} \Delta u^{*}(k+j+1 \mid k)^{T} R \Delta u^{*}(k+j+1 \mid k)+\delta_{y, k}^{*}{ }^{T}\left(\Theta_{T}\right) S_{y} \delta_{y, k}^{*}\left(\Theta_{T}\right)+\delta_{u, k}^{*}{ }^{T} S_{u} \delta_{u, k}^{*}
\end{aligned}
$$

Observe that, since the same input sequence is used and the current estimated state corresponding to the actual model of the plant is equal to the actual state, then the predicted state and output trajectory will be the same as the optimal predicted trajectories at time step $k$. That is, for any $j \geq 1$, we have

$$
x_{T}(k+j \mid k+1)=x_{T}(k+j \mid k)
$$


and

$$
y_{T}(k+j \mid k+1)=y_{T}(k+j \mid k)
$$

In addition, for the true model we have $\tilde{\delta}_{y, k+1}\left(\Theta_{T}\right)=\delta_{y, k}^{*}\left(\Theta_{T}\right)$ and $\tilde{\delta}_{u, k+1}=\delta_{u, k}^{*}$. However, the first of these equalities is not true for the other models, as for these models we have $\hat{x}_{n}(k+1 \mid k+1) \neq x_{n}(k+1 \mid k)$, for $\Theta_{n} \neq \Theta_{T}$.

Now, subtracting (35) from (34) we have

$$
\begin{aligned}
V_{k}^{*}\left(\Theta_{T}\right)-\tilde{V}_{k+1}\left(\Theta_{T}\right) & =\left(y_{T}^{*}(k \mid k)-y_{s p, k}^{*}\left(\Theta_{T}\right)-\delta_{y, k}^{*}\left(\Theta_{T}\right)\right)^{T} Q_{y}\left(y_{T}^{*}(k \mid k)-y_{s p, k}^{*}\left(\Theta_{T}\right)-\delta_{y, k}^{*}\left(\Theta_{T}\right)\right) \\
& +\left(u^{*}(k \mid k)-u_{d e s, k}-\delta_{u, k}^{*}\right)^{T} Q_{u}\left(u^{*}(k \mid k)-u_{d e s, k}-\delta_{u, k}^{*}\right)+\Delta u^{*}(k)^{T} R \Delta u^{*}(k)
\end{aligned}
$$

and, from constraint (25), the following relation is obtained

$$
V_{k+1}^{*}\left(\Theta_{T}\right) \leq \tilde{V}_{k+1}\left(\Theta_{T}\right)
$$

which finally implies

$$
\begin{aligned}
V_{k}^{*}\left(\Theta_{T}\right)-V_{k+1}^{*}\left(\Theta_{T}\right) \geq & \left(y_{T}^{*}(k \mid k)-y_{s p, k}^{*}\left(\Theta_{T}\right)-\delta_{y, k}^{*}\left(\Theta_{T}\right)\right)^{T} Q_{y}\left(y_{T}^{*}(k \mid k)-y_{s p, k}^{*}\left(\Theta_{T}\right)-\delta_{y, k}^{*}\left(\Theta_{T}\right)\right) \\
& +\left(u^{*}(k \mid k)-u_{d e s, k}-\delta_{u, k}^{*}\right)^{T} Q_{u}\left(u^{*}(k \mid k)-u_{d e s, k}-\delta_{u, k}^{*}\right)+\Delta u^{*}(k)^{T} R \Delta u^{*}(k)
\end{aligned}
$$

Since the right hand side of (36) is positive definite, the successive values of the cost will be strictly decreasing and for a large enough time $\bar{k}$, we will have $\left(V_{\bar{k}}^{*}\left(\Theta_{T}\right)-V_{\bar{k}+1}^{*}\left(\Theta_{T}\right)\right)=0$, which proves the convergence of the cost.

The convergence of $V_{k}^{*}\left(\Theta_{T}\right)$ means that, at steady state, the following relations should hold

$$
\begin{gathered}
y_{T}^{*}(\bar{k} \mid \bar{k})-y_{s p, \bar{k}}^{*}\left(\Theta_{T}\right)=\delta_{y, \bar{k}}^{*}\left(\Theta_{T}\right) \\
u^{*}(\bar{k} \mid \bar{k})-u_{d e s, \bar{k}}=\delta_{u, \bar{k}}^{*} \\
\Delta u^{*}(\bar{k})=0
\end{gathered}
$$

At steady state, the state is such that

$$
\hat{x}_{n}(\bar{k})=\left[\begin{array}{c}
y(\bar{k}) \\
\vdots \\
y(\bar{k}+p) \\
\hat{x}_{n}^{s}(\bar{k}) \\
\hat{x}_{n}^{d}(\bar{k})
\end{array}\right]=\left[\begin{array}{c}
y(\bar{k}) \\
\vdots \\
y(\bar{k}) \\
y(\bar{k}) \\
0
\end{array}\right]
$$

where $y(\bar{k})$ is the actual plant output. Note that the state component $\hat{x}_{n}^{d}(\bar{k})$ is null as it corresponds to the stable modes of the system and the input increment is null at steady state. Then, constraint (23) can be written as follows: 


$$
\delta_{y, \bar{k}}^{*}\left(\Theta_{n}\right)=y_{n}^{*}(\bar{k} \mid \bar{k})-y_{s p, \bar{k}}^{*}\left(\Theta_{n}\right)=y(\bar{k})-y_{s p, \bar{k}}^{*}\left(\Theta_{n}\right), \quad n=1, \ldots, L .
$$

This means that, if the output of the true system is stabilized inside the output zone, then the set point corresponding to each particular model will be placed by the optimizer exactly at the output predicted values. As a result, all the output slacks will be null. On the other hand, if the output of the true system is stabilized at a value outside the output zone, then the set-point variable corresponding to any particular model will be placed by the optimizer at the boundary of the zone. In this case, the output slack variables will be different from zero, but they will all have the same numerical value as can be seen from (37).

Now, to strictly prove the convergence of the input and output to their corresponding targets, we must show that slacks $\delta_{u, \bar{k}}$ and $\delta_{y, \bar{k}}\left(\Theta_{T}\right)$ will converge to zero. It is necessary at this point to notice that in the case of zone control the degrees of freedom of the system are no longer the same as in the fixed set-point problem. So, the desired input values may be exactly achieved by the true system, even in the presence of some bounded disturbances. Let us now assume that the system is stabilized at a point where, $\delta_{y, \bar{k}}^{*}\left(\Theta_{1}\right)=\cdots=\delta_{y, \bar{k}}^{*}\left(\Theta_{L}\right) \neq 0$, and $\delta_{u, \bar{k}} \neq 0$. In addition, assume that the desired input value is constant at $u_{d e s, k}$. Then, at time $\bar{k}$ large enough, the cost corresponding to model $\Theta_{n}$ will be reduced to

$$
V_{\bar{k}}^{*}\left(\Theta_{n}\right)=\delta_{y, \bar{k}}^{T}\left(\Theta_{n}\right) S_{y} \delta_{y, \bar{k}}\left(\Theta_{n 1}\right)+\delta_{u, \bar{k}}^{T} S_{u} \delta_{u, \bar{k}}, \quad n=1, \ldots, L,
$$

and constraints (21) and (22) become,

$$
\hat{x}_{n}^{s}(\bar{k})-y_{s p, \bar{k}}\left(\Theta_{n}\right)=\delta_{y, \bar{k}}\left(\Theta_{n}\right), \quad n=1, \cdots, L
$$

and $u(\bar{k}-1)-u_{d e s, \bar{k}}=\delta_{u, \bar{k}}$.

Since $\hat{x}_{n}^{s}(\bar{k})=y(\bar{k}), \quad n=1, \cdots, L$, Eq. (39) can be written as

$$
y(\bar{k})-y_{s p, \bar{k}}\left(\Theta_{n}\right)=\delta_{y, \bar{k}}\left(\Theta_{n}\right), \quad n=1, \cdots, L .
$$

Now, we want to show that if $u(\bar{k}-1)$ and $u_{d e s, \bar{k}}$ are not on the boundary of the input operating range, then it is possible to guide the system toward a point in which the slack variables $\delta_{y, k}\left(\theta_{n}\right)$ and $\delta_{u, k}$ are null, and this point have a smaller cost than the steady state defined above. Assume also for simplicity that $m=1$. Let us consider a candidate solution to problem P2 defined by:

$$
\Delta \bar{u}(\bar{k} / \bar{k})=u_{\text {des }, \bar{k}}-u(\bar{k}-1)=-\delta_{u, \bar{k}}
$$

and

$$
\bar{y}_{s p, \bar{k}}\left(\theta_{n}\right)=y(\bar{k})-B^{s}\left(\theta_{n}\right) \delta_{u, \bar{k}} \quad n=1, \ldots, L
$$

Now, consider the cost function defined in (21), written for time step $\bar{k}$ and the control move defined in (40) and the output set point defined in (41): 


$$
\begin{aligned}
& \bar{V}_{k}\left(\Theta_{n}\right)=\left(\tilde{I}_{y} y(\bar{k})-\tilde{S}_{1}\left(\Theta_{n}\right) \delta_{u, \bar{k}}-\tilde{I}_{y} y_{s p, \bar{k}}\left(\Theta_{n}\right)-\tilde{I}_{y} \delta_{y, \bar{k}}\left(\Theta_{n}\right)\right)^{T} \tilde{Q}_{y} \\
& \quad\left(\tilde{I}_{y} y(\bar{k})-\tilde{S}_{1}\left(\Theta_{n}\right) \delta_{u, \bar{k}}-\tilde{I}_{y} y_{s p, \bar{k}}\left(\Theta_{n}\right)-\tilde{I}_{y} \delta_{y, \bar{k}}\left(\Theta_{n}\right)\right) \\
& +\left(F\left(\Theta_{n}\right)^{m} x^{d}(\bar{k})-\tilde{B}^{d}\left(\Theta_{n}\right) \delta_{u, \bar{k}}\right)^{T} Q_{d}\left(\Theta_{n}\right)\left(F\left(\Theta_{n}\right)^{m} x^{d}(\bar{k})-\tilde{B}^{d}\left(\Theta_{n}\right) \delta_{u, \bar{k}}\right) \\
& +\left(\tilde{I}_{u} u(k-1)-M \delta_{u, \bar{k}}-\tilde{I}_{u} u_{d e s, k}-\tilde{I}_{u} \bar{\delta}_{u, k}\right)^{T} Q_{u}\left(\tilde{I}_{u} u(k-1)-M \delta_{u, \bar{k}}-\tilde{I}_{u} u_{d e s, k}-\tilde{I}_{u} \bar{\delta}_{u, k}\right) \\
& +\left(-\delta_{u, \bar{k}}\right)^{T} \tilde{R}\left(-\delta_{u, \bar{k}}\right)+\bar{\delta}_{y, \bar{k}}\left(\Theta_{n}\right)^{T} S_{y} \bar{\delta}_{y, \bar{k}}\left(\Theta_{n}\right)+\bar{\delta}_{u, \bar{k}}^{T} S_{u} \bar{\delta}_{u, \bar{k}}
\end{aligned}
$$

Now, since the solution defined by $\left(\Delta \bar{u}(\bar{k} / \bar{k}), \bar{\delta}_{y, \bar{k}}\left(\Theta_{n}\right), \bar{\delta}_{u, \bar{k}}\right)$ satisfies constraint (23) and (24), the above cost can be reduced to

$$
\bar{V}_{\bar{k}}\left(\Theta_{n}\right)=\delta_{u, \bar{k}}^{T} S_{\min }^{u}\left(\Theta_{n}\right) \delta_{u, \bar{k}}
$$

where

$$
S_{\min }^{u}\left(\Theta_{n}\right)=\left[\tilde{I}_{y} B^{s}\left(\Theta_{n}\right)-\tilde{S}_{1}\left(\Theta_{n}\right)\right]^{T} \tilde{Q}_{y}\left[\tilde{I}_{y} B^{s}\left(\Theta_{n}\right)-\tilde{S}_{1}\left(\Theta_{n}\right)\right]+\tilde{B}^{d}\left(\Theta_{n}\right)^{T} Q_{d} \tilde{B}^{d}\left(\Theta_{n}\right)+\tilde{R}
$$

Then, if

$$
S_{u}>S_{u}^{\min }\left(\Theta_{n}\right), \quad n=1, \ldots, L,
$$

the cost corresponding to the decision variables defined in (40) and (41) will be smaller than the cost obtained in (38). This means that it is not possible for the system to remain at a point in which the slack variables $\delta_{y, k}\left(\Theta_{n}\right), n=1, \cdots, L$ and $\delta_{u, k}$ are different from zero.

Thus, as long as the system remains controllable, condition (42) is sufficient to guarantee the convergence of the system inputs to their target while the system output will remain within the output zones. $\square$

Observe that only matrix $S_{u}$ is involved in condition (42) because condition (3) assures that the corrected output prediction, i.e. the one corresponding to the desired input values, lies in the feasible zone. In this case, for all positive matrices $S_{y}$, the total cost can be reduced by making the set point variable equal to the steady-state output prediction, which is a feasible solution and produces no additional cost. However, matrix $S_{y}$ is suggested to be large enough to avoid any numerical problem in the optimization solution.

Remark 5: We can prove the stability of the proposed zone controller under the same assumptions considered in the proof of the convergence. Output tracking stability means that for every $\gamma>0$, there exists a $\rho(\gamma)$ such that if $\left\|\bar{x}_{T}(0)\right\|<\rho$, then $\left\|\bar{x}_{T}(k)\right\|<\gamma$ for all $k \geq 0$; where the extended state of the true system $\bar{x}_{T}(k)$ may be defined as follows

$$
\bar{x}_{T}(k)=\left[\begin{array}{c}
y_{T}(k \mid k)-x_{T}^{s}(k) \\
\vdots \\
y_{T}(k+p \mid k)-x_{T}^{s}(k) \\
x_{T}^{s}(k)-y_{s p, k-1}^{*}\left(\Theta_{T}\right) \\
x_{T}^{d}(k) \\
u(k \mid k)-u_{\text {des }, k}
\end{array}\right]
$$


To simplify the proof, we still assume that $m=1$, and suppose that the optimal solution obtained at step $k-1$ is given by $\Delta u_{k-1}^{*}=\Delta u^{*}(k-1 / k-1), y_{s p, k-1}^{*}\left(\Theta_{1}\right), \cdots, y_{s p, k-1}^{*}\left(\Theta_{L}\right)$, $\delta_{y, k-1}^{*}\left(\Theta_{1}\right), \cdots, \delta_{y, k-1}^{*}\left(\Theta_{L}\right)$ and $\delta_{u, k-1}^{*}$.

A feasible solution to problem P2 at time $k$ is given by:

$\Delta \tilde{u}_{k}=0, \tilde{y}_{s p, k}\left(\Theta_{n}\right)=y_{s p, k-1}^{*}\left(\Theta_{n}\right)$, and $\tilde{\delta}_{u, k}$ and $\tilde{\delta}_{y, k}\left(\Theta_{n}\right)$ are such that

$$
\begin{gathered}
u(k-1)+\tilde{I}_{u}^{T} \overbrace{\Delta \tilde{u}_{k}}^{=0}-u_{d e s, k}-\tilde{\delta}_{u, k}=0 \\
\hat{x}_{n}^{s}(k)+\tilde{B}^{s}\left(\Theta_{n}\right) \overbrace{\Delta \tilde{u}_{k}}^{=0}-\tilde{y}_{s p, k}\left(\Theta_{n}\right)-\tilde{\delta}_{y, k}\left(\Theta_{n}\right)=0, \quad n=1, \ldots, L .
\end{gathered}
$$

Since $\Delta \tilde{u}(k \mid k)=0$, we have $u(k \mid k)=u(k-1)$ and from (43) we can write

$$
\tilde{\delta}_{u, k}=u(k \mid k)-u_{\text {des }, k}
$$

For the true system, (44) can be written as follows

$$
x_{T}^{s}(k)-y_{s p, k-1}^{*}\left(\Theta_{T}\right)-\tilde{\delta}_{y, k}\left(\Theta_{T}\right)=0
$$

and consequently, we have the following relations

$$
\tilde{\delta}_{y, k}\left(\Theta_{T}\right)=x_{T}^{s}(k)-y_{s p, k-1}^{*}\left(\Theta_{T}\right)
$$

and

$$
x_{T}^{s}(k)=y_{s p, k-1}^{*}\left(\Theta_{T}\right)+\tilde{\delta}_{y, k}\left(\Theta_{T}\right)
$$

For the feasible solution defined above, the cost defined in (21) can be written for the actual model $\Theta_{T}$ as follows

$$
\begin{aligned}
& \tilde{V}_{k}\left(\Theta_{T}\right)=\left(N_{x} x_{T}(k)-\tilde{I}_{y} y_{s p, k-1}^{*}\left(\Theta_{T}\right)-\tilde{I}_{y} \tilde{\delta}_{y, k}\left(\Theta_{T}\right)\right)^{T} \tilde{Q}_{y}\left(N_{x} x_{T}(k)-\tilde{I}_{y} y_{s p, k-1}^{*}\left(\Theta_{T}\right)-\tilde{I}_{y} \tilde{\delta}_{y, k}\left(\Theta_{T}\right)\right) \\
& \quad+\left(F\left(\Theta_{T}\right)^{m} x_{T}^{d}(k)\right)^{T} Q_{x d}\left(\Theta_{T}\right)\left(F\left(\Theta_{T}\right)^{m} x_{T}^{d}(k)\right) \\
& \quad+\left(\tilde{I}_{u} u(k-1)-\tilde{I}_{u} u_{d e s, k}-\tilde{I}_{u} \tilde{\delta}_{u, k}\right)^{T} Q_{u}\left(\tilde{I}_{u} u(k-1)-\tilde{I}_{u} u_{d e s, k}-\tilde{I}_{u} \tilde{\delta}_{u, k}\right) \\
& \quad+\left(x_{T}^{s}(k)-y_{s p, k-1}^{*}\left(\Theta_{T}\right)\right)^{T} S_{y}\left(x_{T}^{s}(k)-y_{s p, k-1}^{*}\left(\Theta_{T}\right)\right)+\left(u(k \mid k)-u_{d e s, k}\right)^{T} S_{u}\left(u(k \mid k)-u_{d e s, k}\right)
\end{aligned}
$$

Now, using (45), (46) and (47) the cost defined in (48) can be reduced to the following expression

$$
\tilde{V}_{k}\left(\Theta_{T}\right)=\bar{x}_{T}(k)^{T}\left\{C_{1}^{T} \tilde{Q}_{y} C_{1}+C_{2}^{T}\left(F\left(\Theta_{T}\right)^{m}\right)^{T} Q_{d}\left(\Theta_{T}\right)\left(F\left(\Theta_{T}\right)^{m}\right) C_{2}+C_{3}^{T} S_{y} C_{3}+C_{4}^{T} S_{u} C_{4}\right\}
$$

where

$$
C_{1}=\left[\begin{array}{llll}
I_{(p+1) n y} & 0_{(p+1) n y \times n y} & 0_{(p+1) n y \times n d} & 0_{(p+1) n y \times n u}
\end{array}\right]
$$




$$
\begin{aligned}
& C_{2}=\left[\begin{array}{llll}
0_{n d \times(p+1) n y} & 0_{n d \times n y} & I_{n d} & 0_{n d \times n u}
\end{array}\right] \\
& C_{3}=\left[\begin{array}{llll}
0_{n y \times(p+1) n y} & I_{n y} & 0_{n y \times n d} & 0_{n y \times n u}
\end{array}\right] \\
& C_{4}=\left[\begin{array}{llll}
0_{n u \times(p+1) n y} & 0_{n u \times n y} & 0_{n u \times n d} & I_{n u}
\end{array}\right]
\end{aligned}
$$

Thus, the cost defined in (48) can be written as follows:

$$
\tilde{V}_{2, k}\left(\Theta_{T}\right)=\bar{x}_{T}(k)^{T} H_{1}\left(\Theta_{T}\right) \bar{x}_{T}(k),
$$

where $H_{1}=C_{1}^{T} \tilde{Q}_{y} C_{1}+C_{2}^{T}\left(F\left(\Theta_{T}\right)^{m}\right)^{T} Q_{x d}\left(\Theta_{T}\right)\left(F\left(\Theta_{T}\right)^{m}\right) C_{2}+C_{3}^{T} S_{y} C_{3}+C_{4}^{T} S_{u} C_{4}$.

Because of constraint (25), the optimal true cost (that is, the cost based on the true model, considering the optimal solution that minimizes the nominal cost at time $k$ ) will satisfy

$$
V_{k}^{*}\left(\Theta_{T}\right) \leq \tilde{V}_{k}\left(\Theta_{T}\right)
$$

and

$$
V_{k+n}^{*}\left(\Theta_{T}\right) \leq V_{k}^{*}\left(\Theta_{T}\right) \text { for any } n>1 .
$$

By a similar procedure as above and based on the optimal solution at time $k+n$, we can find a feasible solution to Problem P2 at time $k+n+1$, for any $n>1$, such that

$$
\tilde{V}_{k+n+1}\left(\Theta_{T}\right) \leq V_{k+n}^{*}\left(\Theta_{T}\right)
$$

and from the definition of $\tilde{V}_{k+n+1}$ we have

$$
\tilde{V}_{2, k+n+1}\left(\Theta_{T}\right)=\bar{x}_{T}(k+n+1)^{T} H_{1}\left(\Theta_{T}\right) \bar{x}_{T}(k+n+1)
$$

Therefore, combining inequalities (49) to (52) results

$$
\bar{x}_{T}(k+n+1)^{T} H_{1}\left(\Theta_{T}\right) \bar{x}_{T}(k+n+1) \leq \bar{x}_{T}(k)^{T} H_{1}\left(\Theta_{T}\right) \bar{x}_{T}(k), \quad \forall n>1 .
$$

As $H_{1}\left(\Theta_{T}\right)$ is positive definite, it follows that

$$
\left\|\bar{x}_{T}(k+n+1)\right\| \leq \alpha\left(\Theta_{T}\right)\left\|\bar{x}_{T}(k)\right\|, \quad \forall n>1
$$

where

$$
\alpha\left(\Theta_{T}\right)=\left[\frac{\lambda_{\max }\left(H_{1}\left(\Theta_{T}\right)\right)}{\lambda_{\min }\left(H_{1}\left(\Theta_{T}\right)\right)}\right]^{1 / 2} \leq \max _{j}\left[\frac{\lambda_{\max }\left(H_{1}\left(\Theta_{j}\right)\right)}{\lambda_{\min }\left(H_{1}\left(\Theta_{j}\right)\right)}\right]^{1 / 2}
$$

If we restrict the state at time $k$ to the set defined by $\left\|\bar{x}_{T}(k)\right\|<\rho$, then, the state at tine $k+n+1$ will be inside the set defined by 


$$
\left\|\bar{x}_{T}(k+n+1)\right\|<\alpha\left(\Theta_{T}\right) \rho, \quad \forall n>1 .
$$

Which proves stability of the closed loop system, as $\bar{x}_{T}$ will remain inside the ball $\left\|\bar{x}_{T}\right\|<\alpha\left(\Theta_{T}\right) \rho$, where $\alpha\left(\Theta_{T}\right)$ is limited, as long as the closed loop starts from a state inside the ball $\left\|\bar{x}_{T}\right\|<\rho$. Therefore, as we have already proved the convergence of the closed loop, we can now assure that under the assumption of state controllability at the final equilibrium point, the proposed MPC is asymptotically stable.

Remark 6: It is important to observe that even if condition (3) cannot be satisfied by the input target, or the input target is such that one or more outputs need to be kept outside their zones, the proposed controller will still be stable. This is a consequence of the decreasing property of the cost function (inequality (36)) and the inclusion of appropriate slack variables into the optimization problem. When no feasible solution exists, the system will evolve to an operating point in which the slack variables, which at steady state are the same for all the models, are as small as possible, but different from zero. This is an important aspect of the controller, as in practical applications a disturbance may move the system to a point from which it is not possible to reach a steady state that satisfies (3). When this happens, the controller will do the best to compensate the disturbance, while maintaining the system under control.

Remark 7: We may consider the case when the desired input target $u_{d e s, k}$ is outside the feasible set $\vartheta_{u}$ and the case where the set $\vartheta_{u}$ itself is null. If $\vartheta_{u}$ is not null, the input target $u_{\text {des, }, k}$ could be located within the global input feasible set $\vartheta_{0}$, but outside the restricted input feasible set $\vartheta_{u}$. In this case, the slack variables at steady state, $\delta_{u, s s}$ and $\delta_{y, s s}\left(\Theta_{n}\right)$, cannot be simultaneously zeroed, and the relative magnitude of matrices $S_{y}$ and $S_{u}$ will define the equilibrium point. If the priority is to maintain the output inside the corresponding range, the choice must be $S_{y}>>S_{u}$, while preserving $S_{u}>S_{u}^{\min }$. Then, the controller will guide the system to a point in which $\delta_{y, s s}\left(\Theta_{n}\right) \approx 0, n=1, \cdots, L$ and $\delta_{u, s s} \neq 0$. On the other hand, if $\vartheta_{u}$ is null, that is, there is no input belonging to the global input feasible set $\vartheta_{o}$ that simultaneously satisfies all the zones for the models lying in $\Omega$, then, the slack variables $\delta_{y, s s}\left(\Theta_{n}\right), n=1, \cdots, L$, cannot be zeroed, no matter the value of $\delta_{u, s s}$. In this case (assuming that $S_{y} \gg S_{u}$ ), the slack variables $\delta_{y, s s}\left(\Theta_{n}\right), n=1, \cdots, L$, will be made as small as possible, independently of the value of $\delta_{u, s s}$. Then, once the output slack is established, the input slack will be accommodated to satisfy these values of the outputs.

\section{Simulation results for the system with time delay}

The system adopted to test the performance of the robust controller presented here is based on the FCC system presented in Sotomayor and Odloak (2005) and González et al. (2009). It is a typical example of the chemical process industry, and instead of output set points, this system has output zones. The objective of the controller is then to guide the manipulated inputs to the corresponding targets and to maintain the outputs (that are more numerous than the inputs) within the corresponding feasible zones. The system considered here has 2 inputs and 3 outputs. Three models constitute the multi-model set $\Omega$ on which the robust controller is based. In two of these models, time delays were included to represent a possible degradation of the process conditions along an operation campaign. The third model corresponds to the process at the design conditions. The parameters corresponding to each of these models can be seen in the following transfer functions: 


$$
\begin{aligned}
& G\left(\Theta_{1}\right)=\left[\begin{array}{cc}
\frac{0.4515 e^{-2 s}}{2.9846 s+1} & \frac{0.2033 e^{-4 s}}{1.7187 s+1} \\
\frac{1.5 e^{-6 s}}{20 s+1} & \frac{(0.1886 s-3.8087) e^{-3 s}}{17.7347 s^{2}+10.8348 s+1} \\
\frac{1.7455 e^{-6 s}}{9.1085 s+1} & \frac{-6.1355 e^{-5 s}}{10.9088 s+1}
\end{array}\right], \\
& G\left(\Theta_{2}\right)=\left[\begin{array}{cc}
\frac{0.25 e^{-2 s}}{3.5 s+1} & \frac{0.135 e^{-5 s}}{2.77 s+1} \\
\frac{0.9 e^{-3 s}}{25 s+1} & \frac{(0.1886 s-2.8) e^{-4 s}}{19.7347 s^{2}+10.8348 s+1} \\
\frac{1.25 e^{-5 s}}{11.1085 s+1} & \frac{-5 e^{-6 s}}{12.9088 s+1}
\end{array}\right], \\
& G\left(\Theta_{3}\right)=\left[\begin{array}{cc}
\frac{0.7}{1.98 s+1} & \frac{0.5}{2.7 s+1} \\
\frac{2.3}{25 s+1} & \frac{0.1886 s-4.8087}{15.7347 s^{2}+10.8348 s+1} \\
\frac{3}{7 s+1} & \frac{-8.1355}{7.9088 s+1}
\end{array}\right] .
\end{aligned}
$$

In this reduced system, the manipulated input variables correspond to: $u_{1}$ air flow rate to the catalyst regenerator, $u_{2}$ opening of the regenerated catalyst valve, and the controlled outputs are the following: $y_{1}$ riser temperature, $y_{2}$ regenerator dense phase temperature, $y_{3}$ : regenerator dilute phase temperature.

In the simulations considered here, model $\Theta_{1}$ is assumed to be the true model, while model $\Theta_{3}$ represents the nominal model that is used into the MPC cost. In the discussion that follows, unless explicitly mentioned, the adopted tuning parameters of the controller are $m=3, \quad T=1, \quad Q_{y}=0.5 * \operatorname{diag}\left(\begin{array}{lll}1 & 1 & 1\end{array}\right), \quad Q_{u}=\operatorname{diag}\left(\begin{array}{ll}1 & 1\end{array}\right), \quad R=\operatorname{diag}(1 \quad 1)$, $S_{y}=10^{3} * \operatorname{diag}\left(\begin{array}{lll}1 & 1 & 1\end{array}\right)$ and $S_{u}=10^{5 * \operatorname{diag}}\left(\begin{array}{ll}1 & 1\end{array}\right)$. The input and output constraints, as well as the maximum input increments, are shown in Tables 1 and 2.

\begin{tabular}{ccc}
\hline Output & $y_{\min }$ & $y_{\max }$ \\
\hline$y_{1}\left({ }^{\circ} \mathrm{C}\right)$ & 510 & 530 \\
$y_{2}\left({ }^{\circ} \mathrm{C}\right)$ & 600 & 610 \\
$y_{3}\left({ }^{\circ} \mathrm{C}\right)$ & 530 & 590 \\
\hline
\end{tabular}

Table 1. Output zones of the FCC system

\begin{tabular}{cccc}
\hline Input & $\Delta u_{\max }$ & $u_{\min }$ & $u_{\max }$ \\
\hline$u_{1}($ ton $/ \mathrm{h})$ & 25 & 75 & 250 \\
$u_{2}(\%)$ & 25 & 25 & 101 \\
\hline
\end{tabular}

Table 2. Input constraints of the FCC system 
Before starting the detailed analysis of the properties of the proposed robust controller, we find it useful to justify the need for a robust controller for this specific system. We compare, the performance of the proposed robust controller defined through Problem P2, with the performance of the nominal MPC defined through Problem P1. We consider the same scenario described above except for the input targets that are not fully included in the control problem (we consider a target only to input $u_{1}$ by simply making $Q_{u}=\operatorname{diag}\left(\begin{array}{ll}1 & 0\end{array}\right)$ and $S_{u}=10^{5 *} \operatorname{diag}\left(\begin{array}{ll}1 & 0\end{array}\right)$. This is a possible situation that may happen in practice when the process optimizer is sending a target to one of the outputs. Figures 2 and 3 show the output and input responses respectively for the two controllers when the system starts from a steady state where the outputs are outside their zones. It is clear that the conventional MPC cannot stabilize the plant corresponding to model $\Theta_{1}$ when the controller uses model $\Theta_{3}$ to calculate the output predictions. However, the proposed robust controller performs quite well and is able to bring the three outputs to their zones
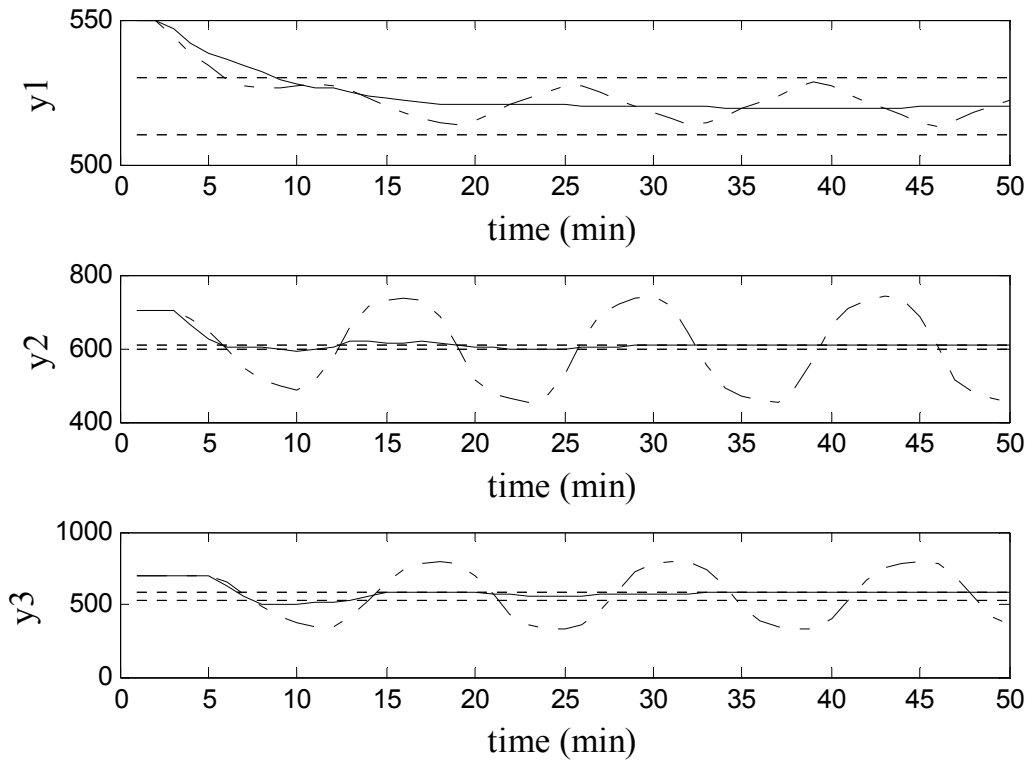

Fig. 2. Controlled outputs for the nominal (- - ) and robust (-) MPC.

We now concentrate our analysis on the application of the proposed controller to the FCC system. As was defined in Eq. (5), each of the three models produces an input feasible set, whose intersection constitutes the restricted input feasible set of the controller. These sets have different shapes and sizes for different stationary operating points (since the disturbance $d_{n}(k)$ is included into Eq. (5), except for the true model case, where the input feasible set remains unmodified as the estimated states exactly match the true states. The closed loop simulation begins at $u_{s s}=\left[\begin{array}{lll}230.5977 & 60.2359\end{array}\right]$ and $y_{s s}=\left[\begin{array}{ll}549.5011 & 704.2756\end{array}\right.$ 690.6233], which are values taken from the real FCC system. For such an operating point, the input feasible set corresponding to models 1, 2 and 3 are depicted in Figure 4 . These sets are quite distinct from each other, which results in an empty restricted feasible input set for the controller $\left(\vartheta_{u}=\vartheta_{u}\left(\Theta_{1}\right) \cap \vartheta_{u}\left(\Theta_{2}\right) \cap \vartheta_{u}\left(\Theta_{3}\right)\right)$. This means that, we cannot find an input that, 
taking into account the gains of all the models and all the estimated states, satisfies the output constraints.
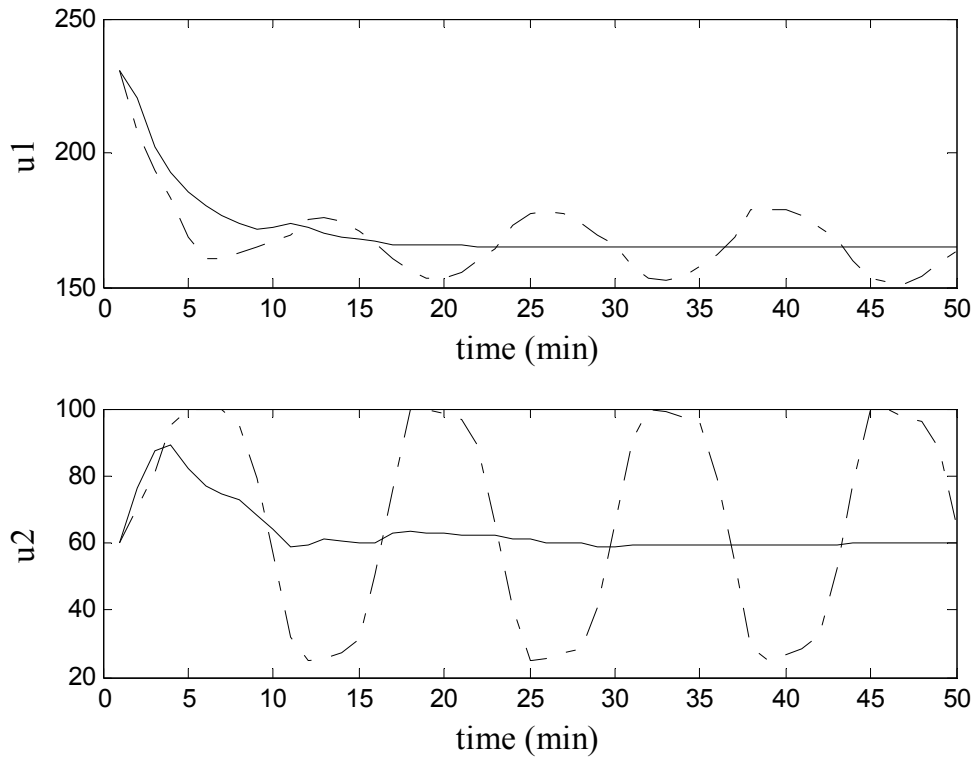

Fig. 3. Manipulated inputs for the nominal (- - ) and robust $(-)$ MPC.

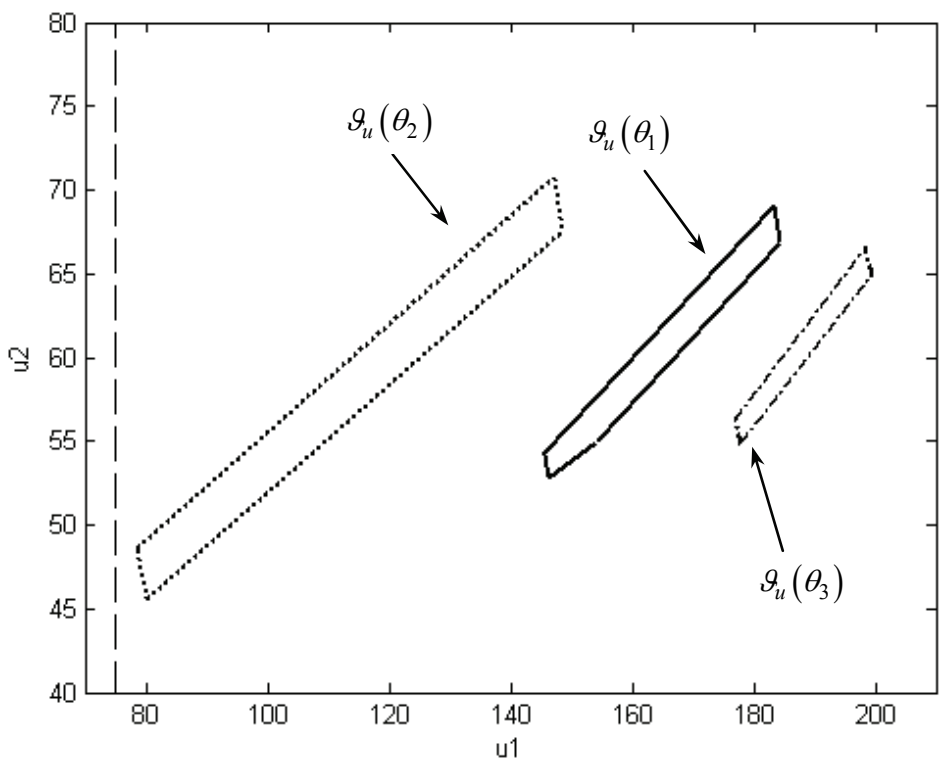

Fig. 4. Input feasible sets of the FCC system 
The first objective of the control simulation is to stabilize the system input at

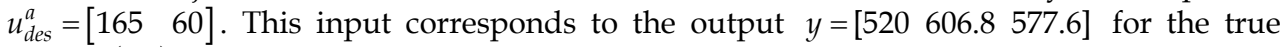
system $\left(\Theta_{1}\right)$, which results in the input feasible sets shown in Figure 5a. In this figure, it can be seen that the input feasible set corresponding to model 1 is the same as in Fig. 4, while the sets corresponding to the other models adapt their shape and size to the new steady state. Once the system is stabilized at this new steady state, we simulate a step change in the target of the input (at time step $k=50 \mathrm{~min}$ ). The new target is given by $u_{\text {des }}^{b}=\left[\begin{array}{ll}175 & 64\end{array}\right]$, and the corresponding input feasible sets are shown in Figure $5 \mathrm{~b}$. In this case, it can be seen that the new target remains inside the new input feasible set $\vartheta_{u}^{b}$, which means that the cost can be guided to zero for the true model. Finally, at time step $k=100 \mathrm{~min}$, when the system reaches the steady state, a different input target is introduced $\left(u_{\text {des }}^{c}=\left[\begin{array}{ll}175 & 58\end{array}\right]\right)$. Differently from the previous targets, this new target is outside the input feasible set $\vartheta_{u}^{c}$, as can be seen in Figure 5c. Since in this case, the cost cannot be guided to zero and the output requirements are more important than the input ones, the inputs are stabilized in a feasible point as close as possible to the desired target. This is an interesting property of the controller as such a change in the target is likely to occur in the real plant operation.
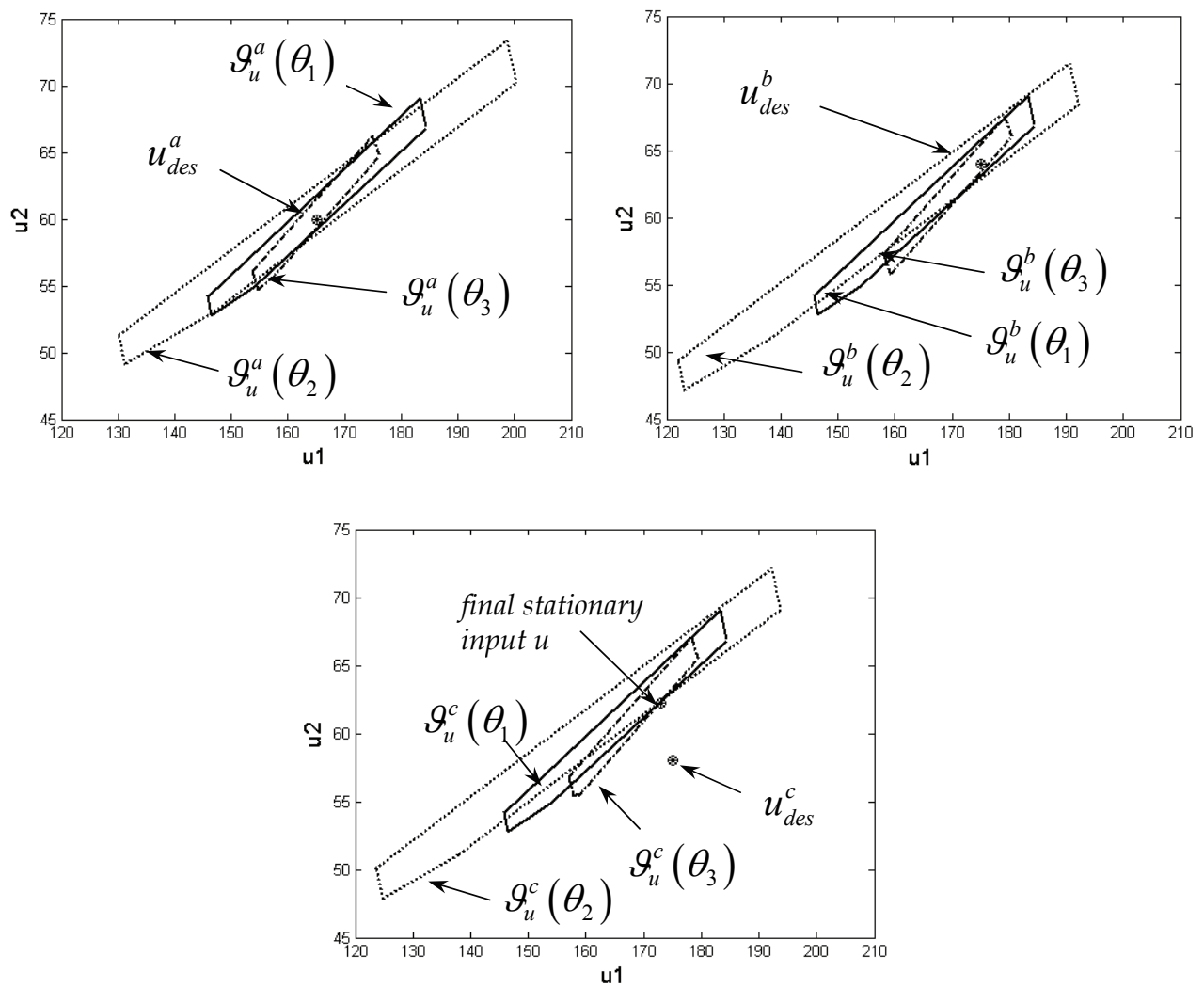

Fig. 5. (a): Initial input feasible sets; (b): Input feasible sets when the first input target is changed; and (c): Input feasible sets when the second input target is changed. 

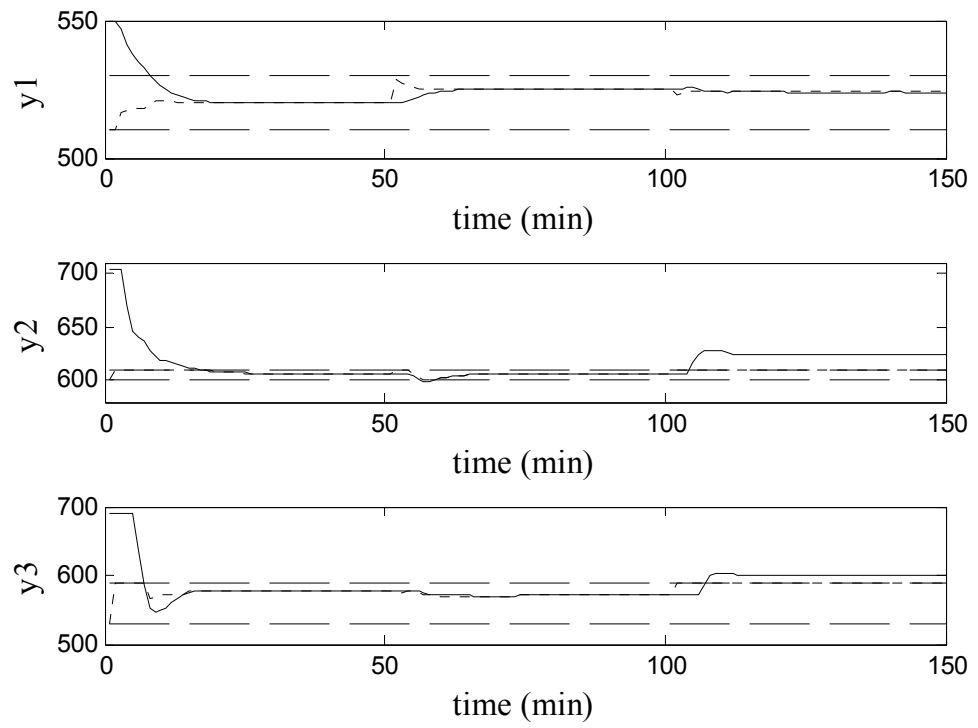

Fig. 6. Controlled outputs and set points for the FCC subsystem with modified input target.
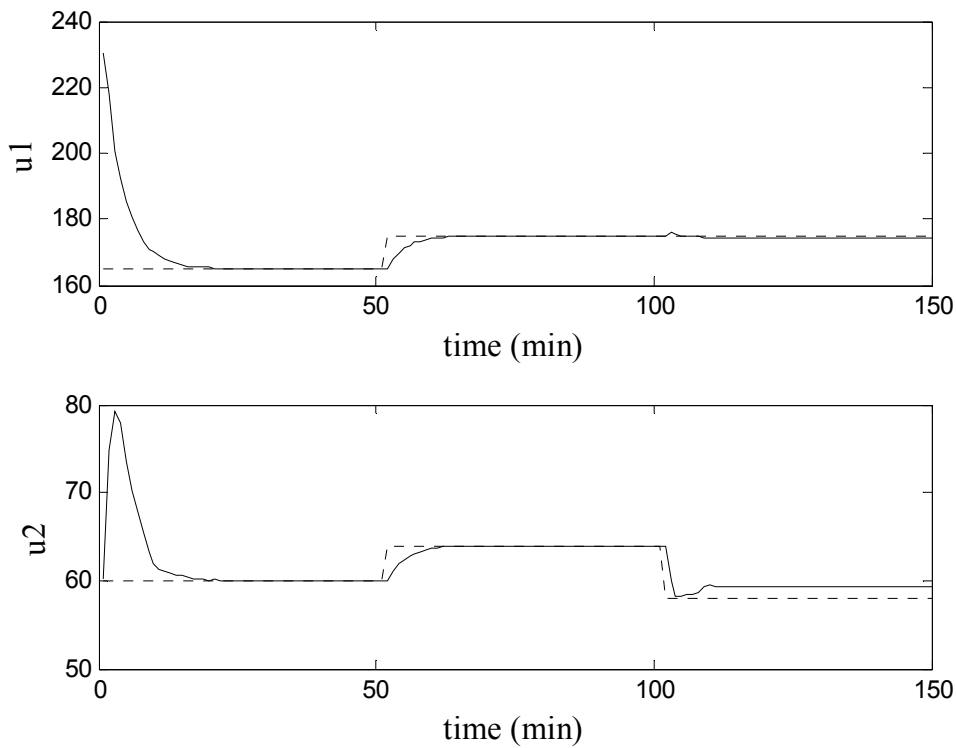

Fig. 7. Manipulated inputs for the FCC subsystem with different input target.

Figure 6 shows the true system outputs (solid line), the set point variables (dotted line) and the output zones (dashed line) for the complete sequence of changes. Figure 7, on the other hand, shows the inputs (solid line), and the input targets (dotted line) for the same sequence. As was established in Theorem 1, the cost function corresponding to the true system is strictly decreasing, and this can be seen in Figure 8. In this figure, the solid line 
represents the true cost function, while the dotted line represents the cost corresponding to model 3. It is interesting to observe that this last cost function is not decreasing, since the estimated state does not match exactly the true state. Note also that in the last period of time, the cost does not reach zero, as the new target is not inside the input feasible set.
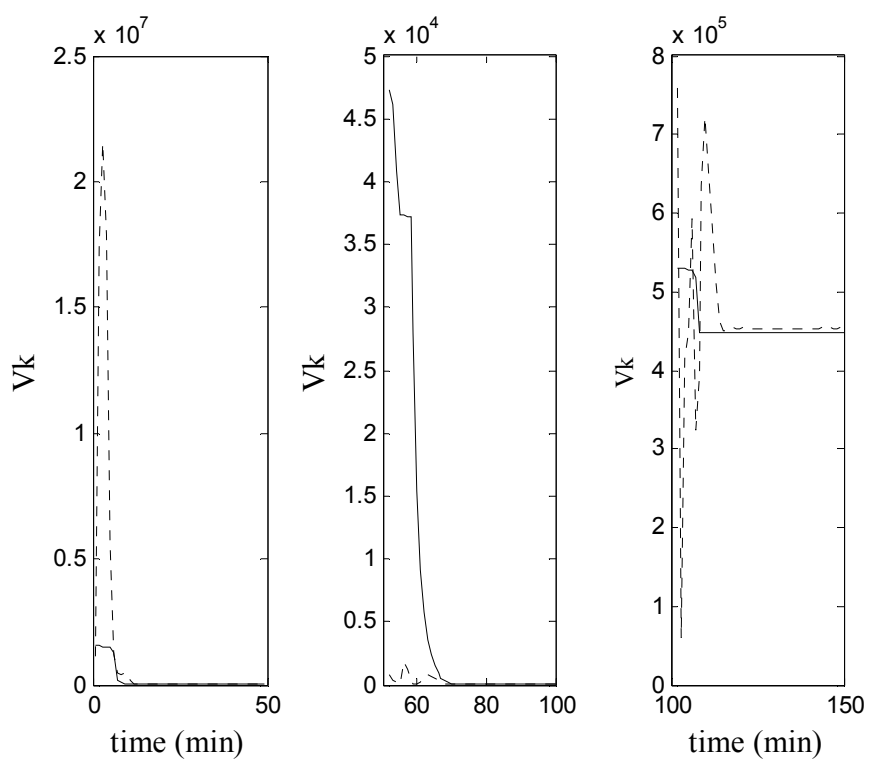

Fig. 8. Cost function corresponding to the true system (solid line) and cost corresponding to model 3 (dotted line).

\begin{tabular}{ccc}
\hline Output & $y_{\min }$ & $y_{\max }$ \\
\hline$y_{1}\left({ }^{\circ} \mathrm{C}\right)$ & 510 & 550 \\
$y_{2}\left({ }^{\circ} \mathrm{C}\right)$ & 400 & 500 \\
$y_{3}\left({ }^{\circ} \mathrm{C}\right)$ & 350 & 500 \\
\hline
\end{tabular}

Table 3. New output zones for the FCC subsystem

Next, we simulate a change in the output zones. The new bounds are given in Table 3 . Corresponding to the new control zones, the input feasible set changes its dimension and shape significantly. In Figure $9, \vartheta_{u}^{a}\left(\Theta_{1}\right)$ corresponds to the initial feasible set for the true model, and $\vartheta_{u}^{d}\left(\Theta_{1}\right), \vartheta_{u}^{d}\left(\Theta_{2}\right)$ and $\vartheta_{u}^{d}\left(\Theta_{3}\right)$ represent the new input feasible sets for the three models considered in the robust controller. Since the input target is outside the input feasible set $\vartheta_{u}^{d}=\vartheta_{u}^{d}\left(\Theta_{1}\right) \cap \vartheta_{u}^{d}\left(\Theta_{2}\right) \cap \vartheta_{u}^{d}\left(\Theta_{3}\right)$, it is not possible to guide the system to a point in which the control cost is null at the end of the simulation time. When the output weight $S_{y}$ is as large as the input weight $S_{u}$, all the outputs are guided to their corresponding zones, while the inputs show a steady state offset with respect to the target $u_{\text {des }}^{a}$. The complete behavior of the outputs and inputs of the FCC subsystem, as well as the output set-points, can be seen in Figures 10 and 11, respectively when $S_{y}=10^{3} * \operatorname{diag}\left(\begin{array}{lll}1 & 1 & 1\end{array}\right)$ and $S_{u}=10^{3} * \operatorname{diag}\left(\begin{array}{ll}1 & 1\end{array}\right)$. The final stationary value of the input is $u=\left[\begin{array}{ll}155 & 84\end{array}\right]$, which represents the closest feasible input value to the target $u_{\text {des }}^{a}$. Finally, Figure 12 shows the control cost of 
the two simulated time periods. Observe that in the last period of time (from $51 \mathrm{~min}$ to 100 $\mathrm{min}$ ) the true cost function does not reach zero since the change in the operating point prevents the input and output constraints to be satisfied simultaneously.

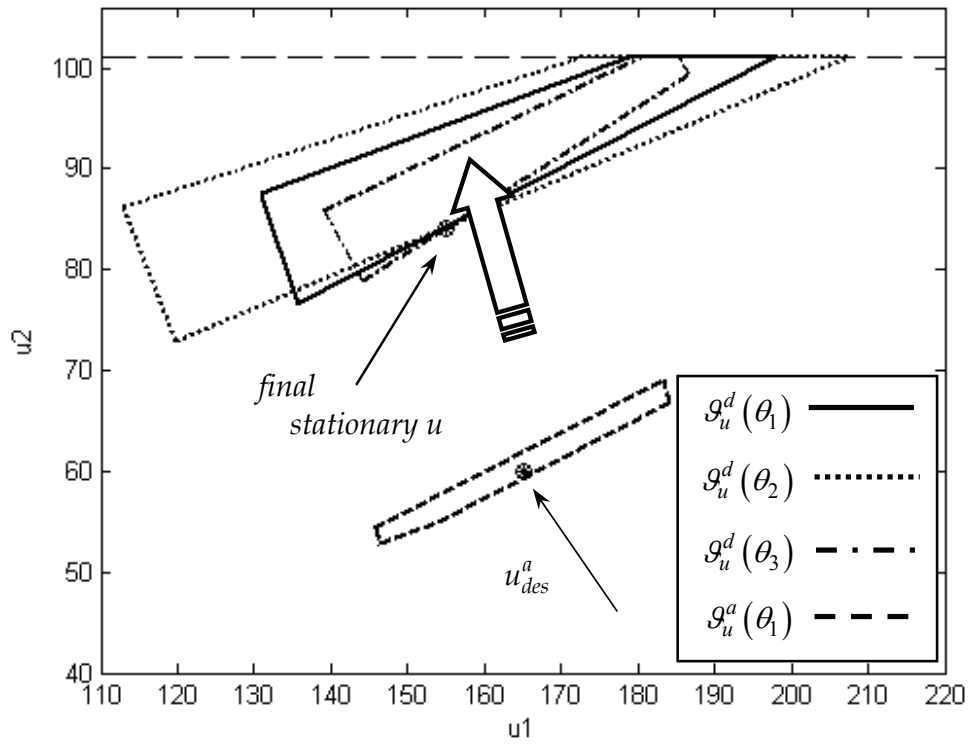

Fig. 9. Input feasible sets for the FCC subsystem when a change in the output zones is introduced.
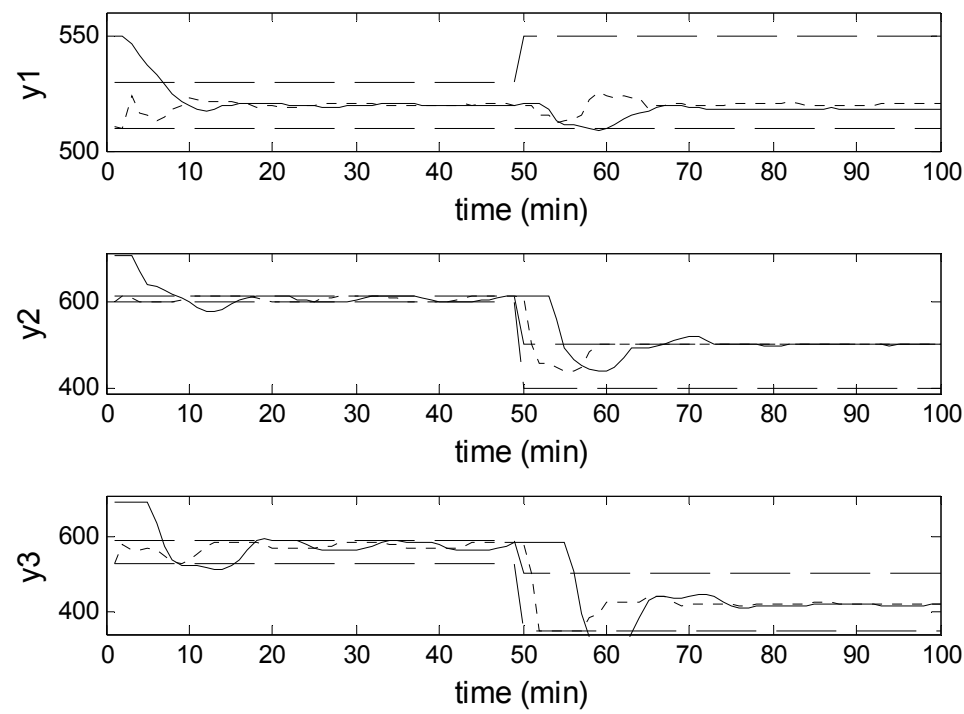

Fig. 10. Controlled outputs and set points for the FCC subsystem with modified zones. 

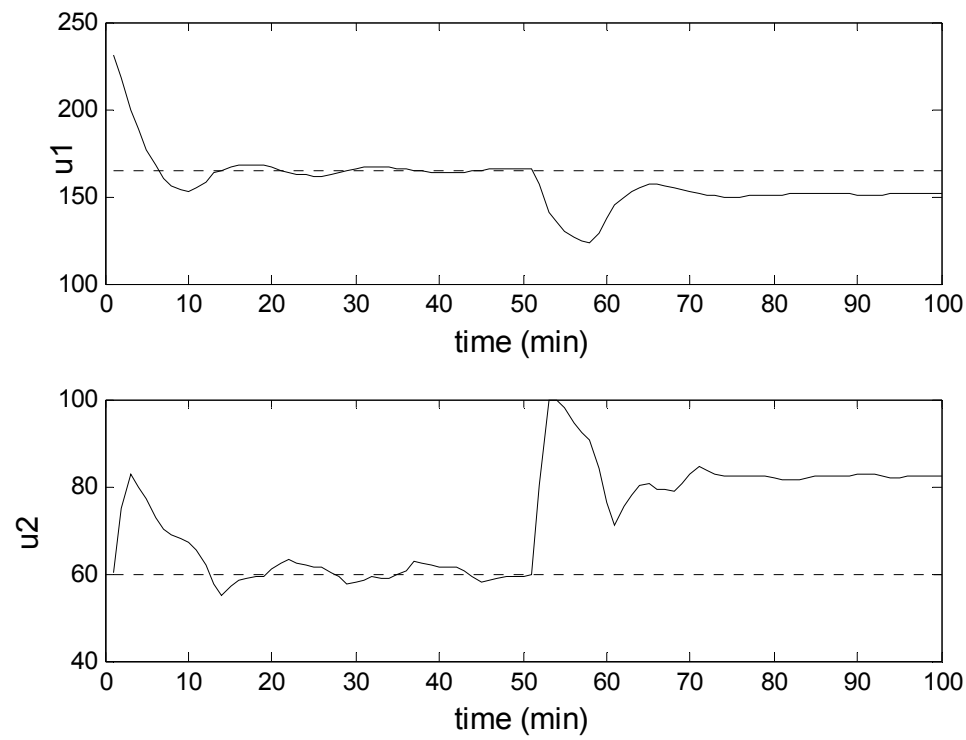

Fig. 11. Manipulated inputs for the FCC subsystem with modified output zones.
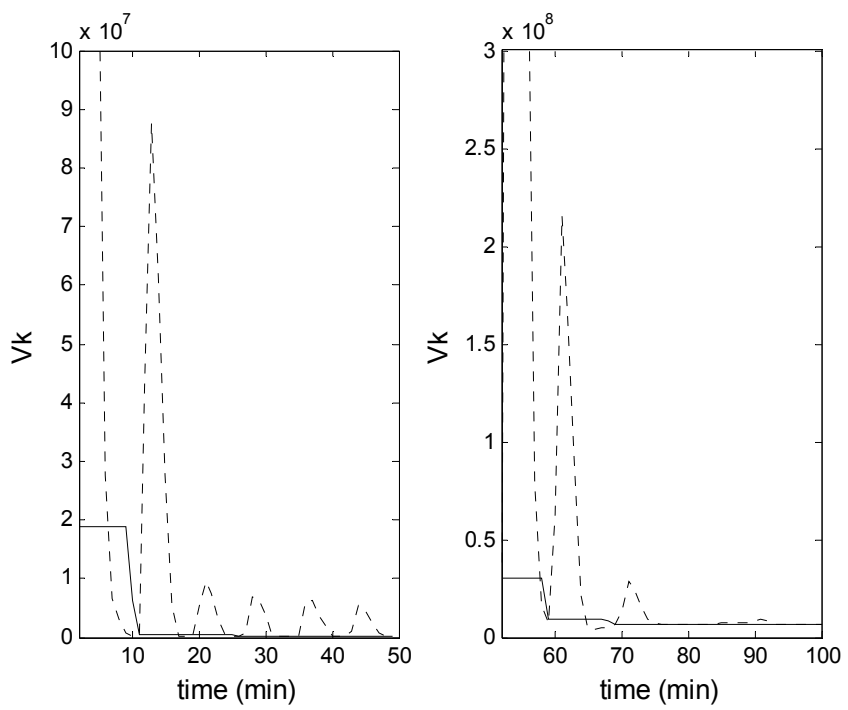

Fig. 12. Cost function for the FCC subsystem with modified zones. True cost function (solid line); Cost function of Model 3 (dotted line).

\section{Conclusion}

In this chapter, a robust MPC previously presented in the literature was extended to the output zone control of time delayed system with input targets. To this end an extended 
model that incorporates additional states to account for the time delay is presented. The control structure assumes that model uncertainty can be represented as a discrete set of models (multi-model uncertainty). The proposed approach assures both, recursive feasibility and stability of the closed loop system. The main idea consists in using an extended set of variables in the control optimization problem, which includes the set point to each predicted output. This approach introduces additional degrees of freedom in the zone control problem. Stability is achieved by imposing non-increasing cost constraints that prevent the cost corresponding to the true plant to increase. The strategy was shown, by simulation, to have an adequate performance for a $2 \times 3$ subsystem of a typical industrial system.

\section{References}

Badgwell T. A. (1997). Robust model predictive control of stable linear systems. International Journal of Control, 68, 797-818.

González A. H.; Odloak D.; Marchetti J. L. \& Sotomayor O. (2006). IHMPC of a HeatExchanger Network. Chemical Engineering Research and Design, 84 (A11), 1041-1050.

González A. H. \& Odloak D. (2009). Stable MPC with zone control. Journal of Process Control, 19, 110-122.

González A. H.; Odloak D. \& Marchetti J. L. (2009) Robust Model Predictive Control with zone control. IET Control Theory Appl., 3, (1), 121-135.

González A. H.; Odloak D. \& Marchetti J. L. (2007). Extended robust predictive control of integrating systems. AIChE Journal, 53 1758-1769.

Kassmann D. E.; Badgwell T. \& Hawkings R. B. (2000). Robust target calculation for model predictive control. AIChE Journal, 45 (5), 1007-1023.

Muske K.R. \& Badgwell T. A. (2002). Disturbance modeling for offset free linear model predictive control. Journal of Process Control, 12, 617-632.

Odloak D. (2004). Extended robust model predictive control. AIChE Journal, 50 (8) 1824-1836.

Pannochia G. \& Rawlings J. B. (2003). Disturbance models for offset-free model-predictive control. AIChE Journal, 49, 426-437.

Qin S.J. \& Badgwell T. A. (2003). A Survey of Industrial Model Predictive Control Technology, Control Engineering Practice, 11 (7), 733-764.

Rawlings J. B. (2000). Tutorial overview of model predictive control. IEEE Control Systems Magazine, 38-52.

Sotomayor O. A. Z. \& Odloak D. (2005). Observer-based fault diagnosis in chemical plants. Chemical Engineering Journal, 112, 93-108.

Zanin A. C.; Gouvêa M. T. \& Odloak D. (2002). Integrating real time optimization into the model predictive controller of the FCC system. Contr. Eng. Pract., 10, 819-831. 


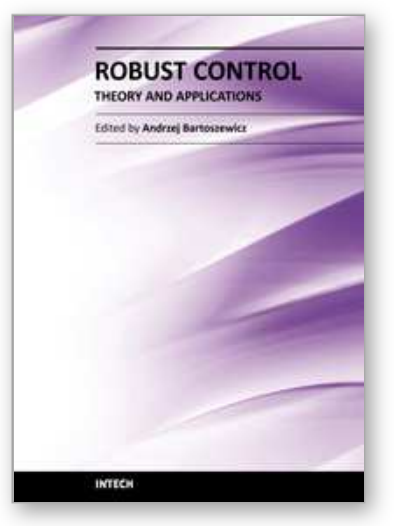

\author{
Robust Control, Theory and Applications \\ Edited by Prof. Andrzej Bartoszewicz
}

ISBN 978-953-307-229-6

Hard cover, 678 pages

Publisher InTech

Published online 11, April, 2011

Published in print edition April, 2011

The main objective of this monograph is to present a broad range of well worked out, recent theoretical and application studies in the field of robust control system analysis and design. The contributions presented here include but are not limited to robust PID, H-infinity, sliding mode, fault tolerant, fuzzy and QFT based control systems. They advance the current progress in the field, and motivate and encourage new ideas and solutions in the robust control area.

\title{
How to reference
}

In order to correctly reference this scholarly work, feel free to copy and paste the following:

Alejandro H. González and Darci Odloak (2011). Robust Model Predictive Control for Time Delayed Systems with Optimizing Targets and Zone Control, Robust Control, Theory and Applications, Prof. Andrzej Bartoszewicz (Ed.), ISBN: 978-953-307-229-6, InTech, Available from:

http://www.intechopen.com/books/robust-control-theory-and-applications/robust-model-predictive-control-fortime-delayed-systems-with-optimizing-targets-and-zone-control

\section{INTECH}

open science | open minds

\section{InTech Europe}

University Campus STeP Ri

Slavka Krautzeka 83/A

51000 Rijeka, Croatia

Phone: +385 (51) 770447

Fax: +385 (51) 686166

www.intechopen.com

\section{InTech China}

Unit 405, Office Block, Hotel Equatorial Shanghai

No.65, Yan An Road (West), Shanghai, 200040, China

中国上海市延安西路65号上海国际贵都大饭店办公楼 405 单元

Phone: +86-21-62489820

Fax: $+86-21-62489821$ 
(C) 2011 The Author(s). Licensee IntechOpen. This chapter is distributed under the terms of the Creative Commons Attribution-NonCommercialShareAlike-3.0 License, which permits use, distribution and reproduction for non-commercial purposes, provided the original is properly cited and derivative works building on this content are distributed under the same license. 